\title{
Graphene Oxide (GO)-based Nanosheets With Combined Chemo/photothermal/Photodynamic Therapy to Overcome Gastric Cancer (GC) Paclitaxel Resistance by Reducing Mitochondria-Derived Adenosine-Triphosphate (ATP)
}

\section{Weihong Guo}

Department of General Surgery, Nanfang Hospital, Southern Medical University

\section{Zhian Chen}

Department of General Surgery, Nanfang Hospital, Southern Medical University

\section{Xiaoli Feng}

Guangdong Provincial Stomatological Hospital

\section{Guodong Shen}

Department of General Surgery, Nanfang Hospital, Southern Medical University

Huilin Huang

Department of General Surgery, Nanfang Hospital, Southern Medical University

\section{Yanrui Liang}

Department of General Surgery, Nanfang Hospital, Southern Medical University

\section{Bingxia Zhao}

Cancer Research Institute, School of Basic Medical Sciences, Southern Medical University

\section{Guoxin Li}

Department of General Surgery, Nanfang Hospital, Southern Medical University

Yanfeng Hu ( $\boldsymbol{\sigma}$ banby@smu.edu.cn )

Department of general surgery, Nanfang Hospital, Southern Medical University, Guangzhou https://orcid.org/0000-0001-5608-3587

\section{Research}

Keywords: Graphene oxide (GO), drug resistance, P-glycoprotein (P-gp), chemo/photothermal (PTT)/ photodynamic (PDT) therapy, mitochondrial respiratory chain

Posted Date: February 23rd, 2021

DOI: https://doi.org/10.21203/rs.3.rs-221992/v1 
License: (c) (i) This work is licensed under a Creative Commons Attribution 4.0 International License. Read Full License 


\section{Abstract}

\section{Background}

Paclitaxel (PTX) has been suggested to be a promising front-line drug for gastric cancer (GC), while Pglycoprotein (P-gp) could lead to drug resistance by pumping PTX out of GC cells. Consequently, it might be a hopeful way to combat drug resistance by inhibiting the out-pumping function of P-gp.

Results

In this study, we developed a drug delivery system incorporating PTX onto polyethylene glycol (PEG)modified and oxidized sodium alginate (OSA) -functionalized graphene oxide (GO) nanosheets (NSs), called PTX@GO-PEG-OSA. Owing to pH/thermal-sensitive drug release properties, PTX@GO-PEG-OSA could induced more obvious antitumor effects on GC, compared to free PTX. With near infrared (NIR)irradiation, PTX@GO-PEG-OSA could generate excessive reactive oxygen species (ROS), attack mitochondrial respiratory chain complex enzyme, reduce adenosine-triphosphate (ATP) supplement for Pgp, and effectively inhibit P-gp's efflux pump function. Since that, PTX@GO-PEG-OSA achieved better therapeutic effect on PTX-resistant GC without evident toxicity (Scheme 1).

\section{Conclusions}

In conclusion, PTX@GO-PEG-OSA could serve as a desirable strategy to reverse PTX's resistance, combined with chemo/photothermal/photodynamic therapy.

\section{Background}

Gastric cancer (GC) is a common malignancy with high morbidity worldwide, especially in East Asia [1, 2]. Most of GC patients are diagnosed with an advanced stage in China, while adjuvant chemotherapy has been preferred for GC's treatment [3]. According to 2019 National Clinical Cancer Network (NCCN), paclitaxel (PTX) is recommended as a front-line treatment (category 1 ) for GC patients $[4,5]$, since PTX could efficiently inhibit spindle function and thus suppress tumor cells' proliferation. Despite an initial antitumor response, multiple drug resistance (MDR) has dramatically compromised PTX's effectiveness in GC cells [6, 7]. Consequently, enhancing the efficacy of PTX with reduced side effects is a clinical problem being discussed.

Currently, drug delivery systems (DDSs) based on nanotechnology provide an opportunity to improve the antitumor efficacy of PTX [8, 9]. Among all materials, graphene oxide (GO) has considerable potential, owing to physically enormous surface area [10], chemically the $\pi-\pi$ stacking interactions originating from the aromatic structure, electrostatic interactions between opposite charges of drug molecules and GO derivatives [11], high thermal conductivity, and plasma membrane traversing capacity [12]. However, low dispersion of GO in protein-rich or salted environments like cell medium and serum, could lead to uncontrollable dose-depended toxicity. Therefore, free GO should be modified via either covalent or 
noncovalent conjugation before using for bio-medical nanocarriers [13]. In our previous research, free GO could induce autophagy-related cell death, revealing its potential toxicity to human beings [14]. As is known, polyethylene glycol (PEG) is one of the most frequently-used reagents in biology owing to its high solubility in organic solvents, minimal toxicity and protein-resistance, and PEG-modified GO (GO-PEG) nanosheets (NSs) showed higher stability and better biocompatibility [13]. Studies have shown that PTXloaded GO-based nano-DDSs have advantages on improving drug solubility and bioavailability, promoting cytotoxicity to cancer cells through enhanced permeability and retention effect (EPR) and reducing toxic side effects, compared to conventional PTX [15]. Meanwhile, pH-responsive smart drug delivery could help release PTX toward the nucleus and then induce more cancer cells death [16]. Oxidized sodium alginate (OSA) is a naturally existent polysaccharide which possesses $\mathrm{pH}$-responsive properties due to the presence of aldehyde groups, and its hydrophilic property could help to avoid drug clearance by the mononuclear phagocyte system post intravenous injection [17-19]. Hence, we assumed that the functionalization of OSA onto GO-PEG might induce a pH-sensitive drug release effect, while GOPEG-OSA nano-DDSs for pH-triggered releasing PTX are yet seldomly reported.

Despite the enhanced absorption and the pH-sensitive release of PTX achieved by smart nano-DDSs, Pglycoprotein (P-gp) could still pump intracellular PTX out of GC cells, reduce drug concentration inside GC cells, and compromised the effectiveness of PTX. Accordingly, inhibiting P-gp's efflux pump function directly would be a promising strategy for the reversal of MDR. Recently, the combination of chemotherapy and near infrared (NIR)-induced phototherapy has been considered to be a valid way against MDR [20], as proper phototherapy could impair P-gp's function [21, 22]. As a result, drugsensitivities revived in MDR cancer cells, namely enhanced absorption of drugs could induce more cell apoptosis at the cancer region. In fact, GO could transfer NIR photon into heat and ROS owing to its broad adsorption spectrum [23, 24], highlighting the photothermal (PTT) and photodynamic (PDT) potentials of GO-based nano-DDSs [25, 26]. In other words, GO-based nano-DDSs could suppress P-gp's efflux pump function directly to achieve better therapeutic effect, under consistent phototherapy triggered by appropriate NIR irradiation. However, few studies reported GO's photo-therapeutic mechanism on inhibiting P-gp's function, and our research will provide new valuable insights into this issue.

In our study, we firstly modified the surface of GO NSs with PEG and OSA, scilicet the pH-sensitive GOPEG-OSA NSs, and then loaded with PTX (PTX@GO-PEG-OSA). The as-prepared NSs were successfully applied to treat PTX-resistant GC cells and achieved excellent chemo/PTT/PDT additive therapeutic effect both in vitro and in vivo. Unlike the reported downregulated expression of P-gp [21], the mechanism of drug resistant reversal was elaborated that PTX@GO-PEG-OSA NSs with NIR-irradiation could induce the depolarization of mitochondrial transmembrane potential (MTP), inhibit the activity of mitochondrial respiratory chain complex enzyme and reduce ATP production, while mitochondrial damage was majorly induced by the generated ROS, instead of hyperthermia. PTX-resistant GC cells were therefore re-sensitive to PTX, since energy supply for P-gp was suppressed.

\section{Materials And Methods}




\subsection{Materials}

GO, polyethylene glycol diamine $\left(\mathrm{NH}_{2}-\mathrm{PEG}-\mathrm{NH}_{2}\right.$; molecular mass:2000), sodium alginate (SA), PTX, 1-(3Dimethylaminopropyl)-3-ethylcarbodiimide hydrochloride (EDC), N-hydroxysuccinimide (NHS) and other reagents were purchased from Aladdin company (China).

\subsection{Synthesis of GO-PEG-OSA NSs}

Sodium hydroxide solution ( $1 \mathrm{~mol} / \mathrm{L})$ was added to nano-GO $(5 \mathrm{mg} / \mathrm{mL})$ dispersion, and the mixtures were maintained with stirring for 1 hour at room temperature. After centrifuging, precipitates were collected, washed with $0.1 \mathrm{~mol} / \mathrm{L}$ hydrochloric acid, centrifuged again until supernatant was neutral.

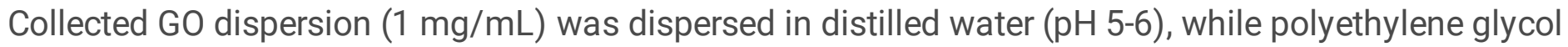
diamine was added and further being stirred for $1 \mathrm{~h}$. After that, $100 \mathrm{mg}$ of EDC and $200 \mathrm{mg}$ of NHS were added, while mixture was stirred for another 12 hours, centrifuged at $6000 \mathrm{rpm}$, and resuspended in distilled water. After purification by repeating dispersion in water and centrifugation, the product was freeze-dried to obtain GO-PEG-NH${ }_{2}$. According to Le-Tien's study [27], $5 \mathrm{~g} \mathrm{SA}$ and $\mathrm{NaClO}_{4}$ solutions were dissolved in $50 \mathrm{~mL}$ deionized water, while the mixtures were stirred for $24 \mathrm{~h}$ in a dark space at room temperature. Then, ethylene glycol was added to the mixtures for $15 \mathrm{~min}$ to stop the reaction. The unreacted $\mathrm{NaClO}_{4}$ was removed by dialysis for 3 days, and the dialysis water was changed 2-3 times a day. After dialysis, the dialysate was freeze-dried to form OSA containing aldehyde groups. OSA was added to a solution of $20 \mathrm{~mL}$ GO-PEG-NH${ }_{2}$ dispersion $(1 \mathrm{mg} / \mathrm{mL})$, and the mixture was stirred for 12 hours at room temperature. The products were purified by dialysis (molecular weight cutoff: 3,500 ) against water for $3 \mathrm{~d}$ and then freeze-dried to yield GO-PEG-OSA.

\subsection{Loading of PTX}

A total of $200 \mu \mathrm{L}$ of PTX solution ( $5 \mathrm{mg} / \mathrm{L}$ ) was added to $2 \mathrm{~mL}$ GO-PEG-OSA or GO-PEG dispersion (5 $\mathrm{mg} / \mathrm{L}$ ), the mixture was stirred at $4{ }^{\circ} \mathrm{C}$ for $12 \mathrm{~h}$ and then centrifuged at $10000 \mathrm{rpm}$ for $20 \mathrm{~min}$. The content of free PTX was measured by collecting the supernatant liquid. The amount of loaded PTX was measured at $227 \mathrm{~nm}$ using HPLC (SHIMADZU, Japan) equipped with Hypersil BDS C18 column (4.6×250 $\mathrm{mm}, 5 \mu \mathrm{m}$, Elite, China). The flow rate of mobile phase (acetonitrile: $\mathrm{H}_{2} \mathrm{O}=55: 45, \mathrm{v} / \mathrm{v}$ ) was $1.0 \mathrm{~mL} / \mathrm{min}$. The PTX loading efficiency (LE) were calculated as following equation:

$\mathrm{LE}(\%)=\frac{\text { Mass of loaded PTX }}{\text { Mass of loaded PTX }+ \text { Mass of feeded " GO - PEG - OSA "conjugates }} \times 100 \%$

\subsection{Characterizations of GO-PEG-OSA NSs}

Fourier transform infrared (FTIR) spectrometer (Vertex 70, Bruker, Germany) was used to obtain the FT-IR spectra. The zeta potential and hydrodynamic diameter was evaluated by Zeta PALS system (Nanobrook Zeta PALS, Brookhaven Instruments Corporation, USA). The morphology was observed by transmission 
electron microscopy (TEM, Hitachi-7650冈Hitachi, Japan) and atomic force microscopy (AFM, NanoscopeMultimode, Veeco, USA). HPLC (Waters 1525, USA) were used to identify the PTX loading and drug release.

\subsection{PTT/PDT effects of PTX@GO-PEG-OSA NSs}

The GO-PEG-OSA and PTX@GO-PEG-OSA NSs were treated by $808 \mathrm{~nm}$ wavelength laser irradiation (1 $\mathrm{W} / \mathrm{cm}^{2}$ or $1.5 \mathrm{~W} / \mathrm{cm}^{2}, 5 \mathrm{~min}$ ), as a thermal probe was used to detect the temperature changes at different time-points, while equivalent amount of PBS with the same laser irradiation was chosen as negative control. The images of temperature changes were recorded by infrared imaging device (ThermaCAMSC3000, Flirsystem Incorporation, USA) at $0.5 \mathrm{~min}$ internals for a total of $5 \mathrm{~min}$.

GO-PEG-OSA and PTX@GO-PEG-OSA NSs were firstly treated with $808 \mathrm{~nm}$ wavelength laser irradiation (1 $\mathrm{W} / \mathrm{cm}^{2}, 5 \mathrm{~min}$ ), and then Singlet Oxygen Sensor Green reagent (Life Technologies, Carlsbad, CA, USA) was used to detect the production of singlet oxygen via a Jasco (Easton, MD, USA) FP-6200 spectrofluorometer.

\subsection{Biocompatibilities of GO-PEG-OSA nanosheets}

\subsubsection{Hemolysis assay}

Blood samples obtained from healthy individuals, were centrifuged and then washed twice with PBS. Then, different concentrations of GO-PEG-OSA were added to blood samples, while the negative and positive control groups were treated with pure PBS solution and Triton X-100 (1\%,v/v), respectively. The mixture was incubated at $37^{\circ} \mathrm{C}$ for 4 hours, followed by centrifuged at 12,000 rpm for 5 min. Finally, the absorbance (optical density [OD]) of the supernatant at a wavelength of $545 \mathrm{~nm}$ was measured.

\subsubsection{In vitro biosafety study}

Normal gastric mucosal epithelial cells (GES-1) were seeded and cultured for $24 \mathrm{~h}$ in Dulbecco's modified Eagle's medium (DMEM) (Gibco) supplemented with $10 \%$ fetal bovine serum (FBS; Gibco). Then cells were incubated with different concentrations of GO-PEG-OSA or GO NSs for $48 \mathrm{~h}$, then cells were analyzed with Cell Counting Kit-8 (CCK-8) assay (Dojido, Tokyo, Japan).

\subsubsection{In vivo biosafety study}

Sprague-Dawley (SD) rats were randomly treated with GO-PEG-OSA $(2 \mathrm{mg} / \mathrm{mL})$ and saline respectively via a tail vein injection. After that, we monitored the function of liver (including aspartate aminotransferase and alanine aminotransferase) and kidney (including blood urea nitrogen and uric acid) for $72 \mathrm{~h}$ by collecting blood samples from SD rats. An overdose of pentobarbital was administered to the rats, and their major organs were harvested for hematoxylin and eosin (H\&E) staining to observe potential damage.

\subsection{Cell uptake and intracellular distribution of PTX@GO-PEG-OSA NSs}


PTX-resistant GC cells (HGC-27/PTX) were seeded and cultured for 24 hours in DMEM with 10\% FBS at $37^{\circ} \mathrm{C}$ and $5 \% \mathrm{CO}_{2}$. Then, cells were treated with PTX@GO-PEG-OSA NSs, with or without pre-treatment of chlorpromazine. After incubation, Inversed Fluorescent Microscope (Olympus, Tokyo, Japan) was applied to observe the cells from 1 to 4 hours. Furthermore, PTX@GO-PEG-OSA NSs' endocytosis was observed by transmission electron microscopy (TEM), ultra-high-performance liquid chromatography (UHPLC) and Confocal Laser Scanning Microscopy (CLSM, Olympus).

\subsection{In vitro antitumor effect of PTX@GO-PEG-OSA NSs}

HGC-27/PTX cells were seeded and cultured for 24 hours in DMEM supplemented with 10\% FBS. Then cells were treated with free PTX, OSA-PEG-OSA or PTX@GO-PEG-OSA NSs separately for 48 h. To evaluate the PTT/PDT of GO-based NSs, the above-treated cells were also treated with or without NIR irradiation ( $\left.1 \mathrm{~W} / \mathrm{cm}^{2}, 5 \mathrm{~min}\right)$, ice incubation or external heating. Then cells were analyzed with CCK-8 assay (Dojido), Annexin-V-FITC/PI assay (KeyGen, Nanjing, China) and 5-ethinyl-2' DNA nucleoside uracil (EdU) assay (KeyGen), according to manual instructions. To explore underlying mechanism, above treated cells were also analyzed with ROS, mitochondrial complex I-V, ATP, Rho123 Assay Kits (all bought from Solarbio, Beijing, China), MTP (KeyGen) and mitochondrial ROS (mitoROS; AAT Bioquest, Wuhan, China).

\subsection{In vivo antitumor effect of PTX@GO-PEG-OSA NSs}

All animal procedures were approved by Animal Ethics Center in Southern Medical University (Certification No. K2020015). Female nude mice (age, 4-5 weeks) were bought from the Experimental Animal Centre of Guangdong Province. To develop tumor-bearing model, HGC-27/PTX cells were harvested and injected into right flank of the nude mice at a density of $5^{\star} 10^{6}$ cells $/ 200 \mu \mathrm{L}$. When tumor volume exceeded $100 \mathrm{~mm}^{3}$, the mice were divided into eight groups, which were treated with saline (negative control), GO-PEG-OSA, free PTX or PTX@GO-PEG-OSA solutions (PTX concentration of 10 $\mathrm{mg} / \mathrm{kg}$ ) via a tail vein injection every $2 \mathrm{~d}$, with or without irradiation of NIR (1 W/cm², $5 \mathrm{~min})$ every $2 \mathrm{~d}$ on tumor sites. Tumor volumes were recorded every day for $21 \mathrm{~d}$. After euthanizing, tumors were excised for data selecting, fixed with formalin for immunohistochemistry (IHC; Tunel, Abcam, USA) and H\&E staining.

\subsection{Western blot analysis}

The RIPA lysis buffer (Beyotime Biotechnology, Shanghai, China) was added into the treated tissue samples for extracting total proteins. Equal quantities of proteins were loaded onto a sodium dodecyl sulfate polyacrylamide gel electrophoresis (SDS-PAGE) gel and transferred onto polyvinylidene difluoride (PVDF) membranes (Millipore, Bedford, Germany). After probing with primary antibodies at $4^{\circ} \mathrm{C}$ overnight, followed by blocking nonspecific antigens with $5 \%$ skimmed milk for 1 hour, the membranes were subsequently washed and incubated with HRP-conjugated secondary antibodies. Finally, the ECL detection system (FDbio, Shenzhen, China) was used to visualize the signals.

\subsection{Statistical analysis}


Mean \pm standard deviation (SD) was used to value the data. Quantitative analysis of the immunofluorescence intensity, western-blotting bands and IHC stained area by aniline was conducted by the Image $J$ program (National Institutes of Health, Bethesda, MD). All experiments were repeated at least 3 times, unless indicated otherwise. The unpaired Student's t-test or the analysis of variance (ANOVA) followed by Scheffe's post hoc test was applied to value the data. When $P<0.05$, significant differences were considered.

\section{Results And Discussion}

\subsection{Synthesis and Characterizations of PTX@GO-PEG-OSA NSs}

Though GO has been widely used in nano-sized DDSs, there are some drawbacks that hamper its applications, such as the tendency to aggregate in protein-rich or salted environments, and its dose dependent-toxicity [14]. To ameliorate this biomaterial, the most efficient approach is to coat the GO surfaces via covalent or noncovalent conjugation. PEG is one of the most frequently used reagents in biology owing to its high solubility in organic solvents, minimal toxicity, and protein resistance [28]. Nevertheless, PTX@GO-PEG NSs lack the ability to respond to external stimuli (such as pH), while these environment-sensitive properties are attractive for customized drug release and precise treatments due to the tumor microenvironments. Alginate is a naturally existing polysaccharide, exhibiting excellent biodegradability, biocompatibility, and water solubility [29]. Oxidized sodium alginate (OSA)-based biomaterials have $\mathrm{pH}$-responsive property now that the presence of aldehyde groups, and its hydrophilic property could help avoid drug clearance by the mononuclear phagocyte system post-intravenous injection $[18,19]$. Consequently, the functionalization of OSA onto GO-PEG might facilitate pH-sensitive drug release effect.

In our research, GO, GO-PEG, GO-PEG-OSA and PTX@GO-PEG-OSA NSs were prepared following the route shown in Scheme 2. As FT-IR spectra demonstrated (Figure 1A), the as-prepared GO showed specific strong and broad peaks at $3400 \mathrm{~cm}^{-1}$ (attributed to hydroxyls and carboxyls), $1620 \mathrm{~cm}^{-1}$ (the skeletal vibrations of $\mathrm{C}=\mathrm{C}$ ), and $1380 \mathrm{~cm}^{-1}$ (the $\mathrm{O}-\mathrm{H}$ deformations of the hydroxyl and epoxy groups) [30]. After PEGylation, GO-PEG NSs showed new peaks at $2950 \mathrm{~cm}^{-1}$ (the skeletal vibrations of $-\mathrm{C}-\mathrm{H}$ ) and $1050 \mathrm{~cm}^{-1}$ (-C-O-), which represented the covalent grafting of PEG chains onto the surface of the GO sheets.

Meanwhile, new peaks at 3200 and $3120 \mathrm{~cm}^{-1}$ (the skeletal vibrations of $-\mathrm{NH}_{2}$ ) was also observed in GOPEG, indicating that free primary amine groups were still available for further conjugation [31].

Furthermore, special peaks at $1610 \mathrm{~cm}^{-1}(-\mathrm{C}=\mathrm{N}$-, characteristic of imine) corresponding to amido-linkages between GO-PEG and OSA appeared after modification with OSA. After PTX's loading, special peaks at $1246 \mathrm{~cm}^{-1}$ (C-N stretching) and $1731 \mathrm{~cm}^{-1}$ (C=0 stretching) corresponding to PTX were also shown [32, 33]. The FI-TR results consistently confirmed the successful synthesis of PTX@GO-PEG-OSA NSs. The completion of surface modification was also demonstrated by the stable hydrodynamic sizes and alternative change of zeta potential (Figure 1B\&C; Supplementary files, Figure S1). It could be seen in the TEM and AFM images that all GO-based products showed special sheet structures, while GO-PEG-OSA 
and PTX@GO-PEG-OSA NSs showed much better dispersibility than unmodified GO (seen in Figure 1E\&F). As-prepared GO-PEG-OSA and PTX@GO-PEG-OSA NSs performed remarkable stability compared to the naked GO nanosheets in both water and FBS (Figure 1D; Supplementary files, Figure S1), which was attributed to the higher zeta potentials in contrast to GO and GO-PEG NSs (Figure 1B\&C; Supplementary files, Figure S1). Furthermore, as GO-based nano-DDSs represent one of the promising nanomedicines to accomplish the photo-therapy, the photothermal conversion ability was tested by exposure of GO-PEG-OSA NSs and PTX@GO-PEG-OSA NSs (GO-PEG-OSA concentration $\sim 0.1 \mathrm{mg} / \mathrm{mL}$ ) under $808 \mathrm{~nm}$ NIR laser irradiation [34]. Significant temperature increase $\left(\sim 8^{\circ} \mathrm{C}\right.$ and $\left.\sim 13^{\circ} \mathrm{C}\right)$ was observed in GO-PEG-OSA group under NIR irradiation (1 W/ $\mathrm{cm}^{2}$ and $\left.1.5 \mathrm{~W} / \mathrm{cm}^{2}\right)$ and PTX@GO-PEG-OSA group showed similar photothermal conversion ability, while free PBS caused only a slight temperature increase (Figure 1G\&H). In addition, higher concentration of GO-PEG-OSA NSs $(\sim 1 \mathrm{mg} / \mathrm{mL})$ was further detected at $1 \mathrm{~W} / \mathrm{cm}^{2}$ and $1.5 \mathrm{~W} / \mathrm{cm}^{2} \mathrm{NIR}$ power intensities, while $\triangle$ Tmax rose to $\sim 14^{\circ} \mathrm{C}$ and $\sim 21^{\circ} \mathrm{C}$ (Supplementary files, Figure S2), indicating that NIR laser can be converted to thermal energy efficiently by GO-PEG-OSA NSs, which was similar to the results of other studies [10,35]. Besides, according to the linear regression curve between the cooling stage and negative natural logarithm of driving force temperature, the photothermal conversion efficiency of GO-PEG-OSA NSs was calculated to be $50.1 \%$. In addition, to determine whether GO-based NSs could produce ROS under NIR laser irradiation, a singlet oxygen sensor was applied to detect the ROS level. As shown in Figure 1I, GO-PEG-OSA and PTX@GOPEG-OSA NSs $(\sim 0.1 \mathrm{mg} / \mathrm{mL})$ exhibited time-dependent production of ROS in deionized water, namely their PDT ability. Therefore, GO-PEG-OSA NSs could be applied for GC's PTT and PDT treatments.

As calculated by HPLC, the PTX loading efficiency (LE) of PTX@GO-PEG-OSA NSs was $8.12 \pm 0.11 \%$. To evaluate the release curve of PTX@GO-PEG-OSA, in vitro release of PTX was monitored in buffers with pH values mimicking those of blood plasma $(\mathrm{pH} \mathrm{7.4)}$ ) and tumor sites $(\mathrm{pH}$ 5.5). As shown in Figure 1J, throughout a 48-hour release period, the release amount of the drug was significantly higher at acidic environment ( $\mathrm{pH}$ 5.5) than neutral ( $\mathrm{pH}$ 7.4). Since GO-PEG-OSA NSs showed PTT capacity, release curve of PTX@GO-PEG-OSA was also monitored in the presence of NIR (1 W/ $\left.\mathrm{cm}^{2}\right)$. As expected, PTX@GO-PEGOSA showed significant increased release rate, indicating their thermal-sensitive drug release abilities. Meanwhile, we detected drug release curve of PTX@GO-PEG NSs, while LE was proved to be $8.00 \pm$ 0.21\%. Throughout a 48-hour release period, PTX release rates in PTX@GO-PEG NSs showed no significant change at pH levels of 5.5 and 7.4 respectively (Supplemental materials, Figure S3). Additionally, the 48h release rate of PTX@GO-PEG-OSA was similar to that of PTX@GO-PEG with a pH value of 5.5, but much slower at pH 7.4 (Figure 1J; Supplemental materials, Figure S3), suggesting that the modification of OSA could effectively reduce the drug leakage in normal tissues. Taken together, we assumed that the amido-linkage between GO-PEG and OSA led to the $\mathrm{pH}$-sensitive drug release, which had a faster degradation rate in acidic environments owing to the Schiff's base structure. This $\mathrm{pH}$ sensitive drug release is beneficial for cancer therapy due to the acidic tumor environments.

\subsection{Biocompatibility of GO-PEG-OSA NSs in vitro and in vivo.}


Good biological safety is an essential prerequisite for nanocarriers [36]. In vitro experiments were firstly performed by incubating GO-PEG-OSA NSs with red blood cells and normal GES-1 cells. The results demonstrated that GO-PEG-OSA NSs did not induce hemolysis at any tested concentrations (as shown in Figure 2A), and CCK-8 assay (Figure 2B) revealed cell viability above $80 \%$ for all concentrations. Besides, immunotoxicities were examined in RAW264.7 murine macrophage-like cells, which implied that GO-PEGOSA NSs $(2 \mathrm{mg} / \mathrm{mL})$ treatment did not induce an inflammatory response in comparison with control group, and LPS significantly increased protrusion formation (Figure 2C). Moreover, the same doses of GO and GO-PEG-OSA were respectively incubated with GES-1 cells, while dramatical cell death was observed in free GO group at dose-dependent manner, compared to that in GO-PEG-OSA group (Supplementary files, Figure S4). All these results strongly indicated that GO-PEG-OSA NSs had perfect in vitro biocompatibilities. To further evaluate their in vivo potential toxicities, saline or GO-PEG-OSA NSs (2 $\mathrm{mg} / \mathrm{mL}$ ) were administered intravenously to SD rats $(\mathrm{n}=5)$. The blood-serum samples were sent for biochemical analyses of clinically relevant indicators, and there were no significant differences among these groups, suggesting that there was no obvious liver or kidney damage apparently (Figure 2D). Additionally, histological analyses of the main organs (brain, kidney, lung, liver and heart) via H\&E staining were performed. No significant pathological changes could be seen between these two groups, which revealed that the GO-PEG-OSA NSs had good biosafety in vivo (Figure 2E).]

\subsection{Cellular uptake and intracellular distribution of PTX@GO-PEG-OSA NSs}

HGC-27/PTX cells were constructed (Supplemental materials, Figure S5) and used for assessment of the as-prepared NSs' cellular uptake and intracellular distribution Bio-TEM images of HGC-27/PTX cells after treatment with PTX@GO-PEG-OSA NSs for 24 h, showed black nanosheets inside cytoplasm, while no NSs were observed in control group (Figure 3A), indicating the cellular uptake of PTX@GO-PEG-OSA NSs. To further observe the endocytosis process, PTX@GO-PEG-OSA were labeled with the fluorescent agent Cy5.5. After incubating with PTX@GO-PEG-OSA from 1 to 4 h, Cy5.5 intensities within HGC-27/PTX cells

were examined by inversed fluorescent microscopy. The fluorescence images (Figure 3B) showed that red fluorescent intensity of Cy5.5-labeled PTX@GO-PEG-OSA NSs increased gradually and reached its peak in 4 hours. In addition, UHPLC results revealed a 4-folds increase of PTX in PTX@GO-PEG-OSA group compared with that for free PTX group (PTX concentrations are both $8 \mu \mathrm{g} / \mathrm{mL}$ ) (Figure 3 C). In order to detect the in vivo distribution of the nanosheets, we constructed the Cy7-loaded PTX@GO-PEG-OSA NSs. After 0-6 h, the mice were imaged using a small animal in vivo fluorescence imaging system (DIGITAL FPRCISION MEDICINE Company, Beijing, China). The in vivo fluorescence images indicate that PTX@GOPEG-OSA NSs could accumulate in the cancer region in $3 \mathrm{~h}$, then gradually enriched in the tumor site from 3 to $24 \mathrm{~h}$. $24 \mathrm{~h}$ later, the tumor-bearing mice were sacrificed, and major organ were collected. And the ex vivo imaging also showed that PTX@GO-PEG-OSA NSs were mostly accumulated in the tumor and liver regions (Supplemental materials, Figure S6).

Several studies have demonstrated that GO-based NSs could enhance cellular uptake of anticarcinogen [37-39]. What's more, Huang et al reported that GO's internalization is mainly dependent on clathrinmediated endocytosis [40], which was a classic endocytosis uptake routine [41]. Consequently, we 
hypothesized that GO-PEG-OSA NSs might also increase the cellular uptake of PTX by the clathrinmediated pathway. To confirm that, we pre-incubated HGC-27/PTX cells with chlorpromazine $(10 \mu \mathrm{g} / \mathrm{mL})$, a specific clathrin protein inhibitor [42, 43]. Fluorescence images and HPLC results consistently showed that the uptake of PTX@GO-PEG-OSA NSs was significantly suppressed in HGC-27/PTX cells

(Supplemental materials, Figure S7). Taken together, our designed PTX@GO-PEG-OSA NSs could effectively facilitate the intracellular delivery efficiency of PTX to GC cells, mostly dependent on clathrinmediated pathway. To further observe the intracellular distribution, HGC-27/PTX cells lysosomes were firstly labeled with green lysotracker probes. After incubation with PTX@GO-PEG-OSA NSs for 24 h, CLSM images showed that the location of PTX@GO-PEG-OSA largely overlapped with that of the lysosomes, and this phenomenon suggested that PTX@GO-PEG-OSA might have entered the lysosomes (Figure 3D).

\subsection{In vitro chemo-therapeutic effect of PTX@GO-PEG-OSA NSs}

To assess the chemo-therapeutic effect of our as-prepared NSs, HGC-27/PTX cells were incubated with different concentrations of PTX@GO-PEG-OSA NSs. According to the CCK-8 assays (Figure 4A), GO-PEGOSA NSs showed relatively nontoxic to GC cells, as the rates of cell viability exceeded $90 \%$ after incubation. To date, it could be seen that PTX@GO-PEG-OSA groups showed more significant therapeutic effects on HGC-27/PTX cells than a parallel dose of free PTX. To further detect the chemo-therapeutic effect, HGC-27/PTX cells were incubated with PTX@GO-PEG-OSA NSs $(8 \mu \mathrm{g} / \mathrm{mL})$ for $24 \mathrm{~h}$, and then analyzed by EdU and apoptosis assay.

EdU dye is a kind of thymidine nucleoside analogues, which could specifically insert into DNA molecules of rapid proliferation cells [44]. After conjugated reactions with EdU, less EdU-positive cells were found in PTX@GO-PEG-OSA and PTX groups, compared to GO-PEG-OSA and blank groups, while PTX@GO-PEGOSA showed least EdU-positive cells (Figure 4B). The apoptosis rates determined by flow cytometry (Figure 4C) demonstrated that PTX@GO-PEG-OSA induced 1.5-folds higher levels of total apoptosis (12\%) than free PTX (7\%), and 2-folds higher total apoptosis than blank and GO-PEG-OSA (5\%). Actually, Cleaved caspase-3, Bax and p53 are all of pro-apoptotic protein that are up-regulated early in a sequence of events associated with programed cell death, especially apoptosis [45]. Consistent with flow cytometry data, the expression of apoptosis-related proteins (including cleaved caspase-3, Bax and p53) remarkably increased in PTX-treated and PTX@GO-PEG-OSA-treated cells, while the anti-apoptosis marker (Bcl-2) was contrarily depleted in comparison to the GO-PEG-OSA treated and control cells (Figure 4D; Supplemental materials, Figure S8). Although the expression levels of the apoptosis marker proteins in PTX-treated cells were upregulated, the changes were still significantly lower than those in PTX@GO-PEG-OSA-treated cells. These results consistently revealed that PTX@GO-PEG-OSA possessed the most remarkable antiproliferation properties. Apart from that, PTX@GO-PEG-OSA NSs were supposed to enter the lysosomes (Figure 3D), which are degradative organelles with a much lower pH microenvironment [46]. We hypothesized that the release of PTX might accelerate and therapeutic effect of PTX@GO-PEG-OSA might be superior to free PTX due to lysosomes. To verify the hypothesis, HGC-27/PTX cells were pre-incubated with $\mathrm{NH}_{4} \mathrm{Cl}$, which could neutralize the acidic microenvironment. Compared with the PTX@GO-PEG-OSA- 
treated cells, the $\mathrm{NH}_{4} \mathrm{Cl}$-treated cells showed significantly higher cell survival rates (Figure 4E), confirming the $\mathrm{pH}$-triggered drug release is useful for enhancing antitumor effect.

\subsection{In vitro chemo/PTT/PDT effects of PTX@GO-PEG-OSA NSs}

As it has been reported, PTT/PDT combined effect could increase the sensitivity of drug-resistant cancer cells to chemotherapeutic agents, thus facilitating the ability to overcome the drug resistance[47, 48]. In addition, the additive chemo/PTT/PDT effects of PTX@GO-PEG-OSA NSs $(8 \mu \mathrm{g} / \mathrm{mL})$ was evaluated. CCK8 assays revealed that PTX@GO-PEG-OSA with NIR (refer to chemo/PTT/PDT) caused 2-folds changes higher cell toxicity ( 70\%) than PTX@GO-PEG-OSA without NIR (refer to chemo, 30\%) and GO-PEG-OSA with NIR (refer to PTT/PDT, 22\%) treatment. Meanwhile, NIR and GO-PEG-OSA group showed significant no viability change, compared to blank group (Figure 5A). EdU assay demonstrated that GO-PEG-OSA with NIR, PTX@GO-PEG-OSA and PTX@GO-PEG-OSA with NIR significantly induced less EdU-positive cell rates than GO-PEG-OSA did, while PTX@GO-PEG-OSA with NIR showed the most obvious anti-

proliferation properties (Figure 5B). The apoptosis rates determined by flow cytometry (Figure 5C) implied that PTX@GO-PEG-OSA with NIR induced 2-folds higher levels of total apoptosis (27\%) than GO-PEG-OSA with NIR (12\%), PTX@GO-PEG-OSA (13\%) and GO-PEG-OSA (5\%). Consistently, the expression of apoptosis proteins (including cleaved caspase-3, Bax and p53) also remarkably increased in GO-PEGOSA+NIR, PTX@GO-PEG-OSA and PTX@GO-PEG-OSA+NIR groups, while the anti-apoptosis marker (Bcl-2) was contrarily exhausted (Figure 5D;Supplemental materials, Figure S9). Among all the groups, the additive therapeutic PTX@GO-PEG-OSA+NIR group showed vastly obvious changes. Totally, both cell viabilities and apoptosis rates showed that PTX@GO-PEG-OSA with NIR induced a combination of chemo/PTT/PDT effects, namely increasing motility in HGC-27/PTX cells, a result that was much better than the results of single chemotherapy or single photo-therapeutic effect (Figure 5A).

\subsection{PTT/PDT effects induced by GO-PEG-OSA NSs could combat drug resistance by reducing mitochondria-derived ATP}

Although the as-prepared PTX@GO-PEG-OSA NSs could enhance the sensitivity to PTX in HGC-27/PTX cells with the assistance of PTT/PDT additive therapy, the underlying mechanism remains unknown. To clarify the roles of PTT and PDT on increasing PTX sensitivity, PTX@GO-PEG-OSA (containing $8 \mu \mathrm{g} / \mathrm{mL}$ of PTX and $\sim 100 \mu \mathrm{g} / \mathrm{mL}$ of GO-PEG-OSA) or GO-PEG-OSA ( 100 $\mu \mathrm{g} / \mathrm{mL}$ of GO-PEG-OSA) groups were further applied on the surface of ice while being irradiated with NIR light, in order to control the temperature below $10^{\circ} \mathrm{C}$ and minimize the effect of NIR-induced PTT ablation. Furthermore, GO-PEG-OSA with NIR group was monitored with an infrared camera, and the temperature could be raised to about $43^{\circ} \mathrm{C}$. An external heating group was accordingly set with temperature of $43^{\circ} \mathrm{C}$ to simulating the PTT, using a cell culture incubation. Whereupon, HGC-27/PTX cells were randomly divided into seven groups, including blank (control), GO-PEG-OSA+NIR (refer to PTT\&PDT), External heating (refer to PTT), GO-PEGOSA+NIR+Ice (refer to PDT), PTX@GO-PEG-OSA (refer to chemo), PTX@GO-PEG-OSA+External heating (refer to chemo\&PTT), PTX@GO-PEG-OSA+NIR+Ice (refer to chemo\&PDT). From CCK-8 results, we found that both GO-PEG-OSA+NIR and External heating groups could induce $20 \%$ cell death, while no

Page 12/32 
significant cell death was shown in GO-PEG-OSA+NIR+Ice group (Supplemental materials, Figure S10). Subsequently, chemo\&PTT and chemo\&PDT effects were also carried out. CCK-8 assays revealed that PTX@GO-PEG-OSA caused 30\% cell toxicity, and PTX@GO-PEG-OSA+External heating (chemo\&PTT) showed $\sim 55 \%$ cell death. Furthermore, PTX@GO-PEG-OSA+NIR+Ice (chemo\&PDT) also showed $46 \%$ cell death (Supplemental materials, Figure S10). Thus, we concluded that PTT\&PDT caused by our designed GO-based NSs could induce GC cell death, where hyperthermia was the key factor on killing tumor cells. Apart from this, the addition of PDT effect exerted super-additive therapeutic efficiency in PTX@GO-PEGOSA groups.

MDR is highly associated with transmembrane efflux pumps such as P-gp, which belongs to the adenosine triphosphate (ATP)-binding cassette (ABC) transporter superfamily $[49,50]$. Some studies have reported that the PDT effect could downregulate the expression level of P-gp, and thus increase the sensitivity of drug-resistant cancer cells $[51,52]$. Though it was also identified that compared to normal HGC-27 cells, P-gp was overexpressed and downregulation of P-gp could enhance sensitivity of PTX in HGC-27/PTX cells (Supplemental materials, Figure S11), it is very surprising that there were no significant differences in P-gp expression level when we treated HGC-27/PTX cells with PTX@GO-PEG-OSA or GOPEG-OSA with or without the presence of NIR irradiation (Supplemental materials, Figure S12 A\&B). That is to say, some other factors might lead to the additive therapy effect rather than the reported inhibition of P-gp proteins' expression. Considering P-gp is a transmembrane glycoprotein with a molecular weight of $170 \mathrm{KD}$ and works as an energy-dependent "drug pump", they could bind to ATP, acquire energy to pump intracellular drugs out of the cell, reduce the drug concentration in the cell and result in drug resistance $[53,54]$. Additionally, intracellular ATP production mostly depends on the mitochondrial respiratory chain complex enzymatic activities [55]. Therefore, reducing mitochondria-derived ATP in cancer cells should be another effective tactic to overcome MDR [56]. As it has been reported, ROS is an inevitable product of cell metabolism, while phototherapeutic effect could cause high levels of intracellular ROS and lead to mitochondrial damage. Based on these, we hypothesized that these GO-based NSs with NIR irradiation might stimulate the generation of ROS inside HGC-27/PTX cells, trigger inner mitochondrial damage by means of its phototherapeutic effects. As a result, ATP can't be synthesized by ATP synthase and the efflux pump ability of P-gp should become lost due to the lack of energy supply. To verify this, we firstly treated the HGC-27/PTX cells with our designed GO-PEG-OSA $(\sim 0.1 \mathrm{mg} / \mathrm{mL})$ NSs with NIR. After the intervention, ROS generation was found to be significantly increased in PTX@GO-PEG-OSA+NIR or GOPEG-OSA+NIR groups, whereas no significant changes were observed in either GO-PEG-OSA, PTX@GOPEG-OSA or NIR laser irradiation alone groups, compared to blank group (Figure 6A; Supplemental materials, Figure S13). Meanwhile, significant ROS (green) was observed in GO-PEG-OSA with NIR group, and ROS was partly overlapped with mitochondria (red), compared to blank and GO-PEG-OSA groups (Supplemental materials, Figure S14). As a result, GO-PEG-OSA plus NIR inducing ROS could still attack mitochondria, even though most of the GO-PEG-OSA NSs might have entered the lysosomes (Figure 3D). Consistently, we found that the treatment of GO-PEG-OSA with NIR did occasion a sharp decrease of MTP (Figure 6B), indicating the damage of mitochondria in HGC-27/PTX cells. Accompanied strong production of ROS in mitochondria was also observed (Figure 6C), which could react on the total growth of ROS in 
cells and the vicious circle of mitochondrial damage in turn. We further investigated the relative contribution of PTT on ROS generation and mitochondrial damage, while our results showed that PTT alone did not significantly increase ROS or injury mitochondria ((Supplemental materials, Figure S14, 1517). Taken together, our research indicated that PDT seemed to play a powerful role on ROS generation and mitochondrial damage, thus help exert super-additive therapeutic efficiency of PTX@GO-PEG-OSA NSs. However, the mechanism of ROS suppressing ATP production in mitochondria, is still not fully explained.

Generally, the mammalian mitochondrial electron transport chain (ETC) includes complexes I-IV [57]. There are two electron transport pathways in the ETC, including NADH-dependent complex I/III/IV and succinic acid-dependent complex II/III/IV. In the ETC, ATP could be normally synthesized by ATP synthase (also called complex V) [58]. However, the transform process from complexes would be suppressed when excessive ROS was produced in mitochondria, and the concentration gradient of protons across the inner membrane of mitochondria would be subsequently minimized $[59,60]$. In our research, the activities of complexes I/III/IV/V were consistently decreased in HGC-27/PTX cells with GO-PEG-OSA plus NIR treatment, while no significant change was observed on complex II, indicating GO-PEG-OSA plus NIR mainly injures NADH-dependent ETC. As the damage of mitochondria complexes, the generation of ATP was evidently suppressed (Figure 6I). Subsequently, more Rho123 was sequestered in HGC-27/PTX cells (Figure 6J), implying the efflux pump dysfunction of P-gp. Oligomycin (an inhibitor of ATP synthase) was also applied for $12 \mathrm{~h}$ to check the function of P-gp, while similar decrease of ATP and loss-function of Pgp were observed in HGC-27/PTX cells (Figure 6I\&J). These results suggested mitochondrial-complexes I/III/IV/V chain was damaged in HGC-27/PTX cells treated with GO-PEG-OSA plus NIR, resulting in reducing ATP production and compromising the function of P-gp pumps in the MDR GC cells. In short, we found GO-PEG-OSA ( 0.1 mg/mL) with NIR irradiation $\left(1 \mathrm{~W} / \mathrm{cm}^{2}, 5 \mathrm{~min}\right)$ maintained an appropriate temperature $\left(43^{\circ} \mathrm{C}\right)$, to which normal cells are generally tolerant and could prevent normal organs near the tumor from damaging when a comparatively high temperature was applied. On the other hand, the excessive produced ROS inside PTX-resistant GC cells, could efficiently depolarize mitochondrial membrane potential, inhibit mitochondrial respiratory chain complexes (I/III/IV/V) enzyme activities, reduce the amount of ATP available for P-glycoprotein (P-gp) and finally reverse gastric cancer drug resistance.

\subsection{Inhibited production of ROS could increase ATP supply for P-gp efflux pumps and compromise MDR- reversal abilities of GO-PEG-OSA in HGC-27/PTX cells}

Actually, the mitochondrial-dependent damage could be triggered via a range of exogenous and endogenous stimuli, such as oxidative stress, ischemia and DNA damage [61, 62]. Meanwhile, previous studies had proved that photo-therapy could induce the outbreak of ROS $[63,64]$, following by the activation of oxidative stress and the mediation of mitochondrial damage in cancer cells $[65,66]$. In order to verify whether GO-PEG-OSA plus NIR causes mitochondrial dysfunction by producing ROS, HGC27/PTX cells were pretreated with N-Acetyl-L-cysteine (NAC), an anti-oxidant containing sulfhydryl, before being treated with GO-PEG-OSA plus NIR. With the addition of NAC, ROS production was majorly reduced 
(Figure 7A), while MTP and mitoROS in HGC-27/PTX cells were consistently recovered (Figure 7B\&C). Furthermore, a sharp increase of total ATP in HGC-27/PTX cells was also observed (Figure 7D). We subsequently checked the function of P-gp in HGC-27/PTX cells treated with GO-PEG-OSA plus NIR. As shown in Figure 7E, less Rho123 was sequestered in HGC-27/PTX cells, indicating that the function of Pgp efflux pumps was indeed rescued. Exogenous ATP was also applied as positive control group to check the function of P-gp, while similar increase of ATP and regain-function of P-gp were observed in HGC27/PTX cells (Figure 7D\&E). These results suggested it's efficiently promoting generation of ROS that the key of GO-PEG-OSA plus NIR increasing mitochondrial-complexes chain damage and reducing ATP production in HGC-27/PTX cells.

To further explore the relationship of excessive ROS generated by GO-PEG-OSA plus NIR-treatment and HGC-27/PTX cells' drug resistance, we detected the additive chemo/PDT-therapeutic effect of PTX@G0PEG-OSA NSs $(8 \mu \mathrm{g} / \mathrm{mL})$ under NIR irradiation $\left(1 \mathrm{~W} / \mathrm{cm}^{2}, 5 \mathrm{~min}\right)$. The CCK-8 assays (Figure 7F) revealed that PTX@GO-PEG-OSA plus NIR caused obvious cell death to HGC-27/PTX cells. After the addition of exogenous NAC (experimental group) or ATP (positive control), cell viabilities were dramatically increased. EdU assay demonstrated that PTX@GO-PEG-OSA plus NIR significantly reduced EdU-positive cell rates, while additional exogenous NAC and ATP could partly increase EdU-positive cell rates (Figure 7G). Consistently, the apoptosis rates determined by flow cytometry (Figure 7H) expounded that PTX@GO-PEG-OSA plus NIR induced 2-folds higher levels of total apoptosis $(27 \%)$ than exogenous NAC (15\%) and exogenous ATP (16\%) groups.

In a word, these results elaborated that stimulating generation of ROS was an essential part for our designed GO-based NSs, in depleting mitochondrial-derived ATP and further increasing PTX sensitivity in HGC-27/PTX cells.

\subsection{In vivochemo/PTT/PDT effects of PTX@GO-PEG-OSA.}

Moreover, to analyze the anti-tumor effect of PTX@GO-PEG-OSA in vivo, we constructed eight groups of tumor-bearing mice, and treated them with saline (negative control), GO-PEG-OSA, PTX or PTX@GO-PEGOSA, with or without NIR irradiation. After the 21-day posttreatment, we found that tumor weights and volumes in PTX@GO-PEG-OSA plus NIR irradiation group were less than those of the other seven groups (Figure 8A\&B\&C), while there were no differences of tumor weights and volumes among saline group, saline plus NIR and GO-PEG-OSA groups, showing that GO-PEG-OSA or NIR themselves could not inhibit tumor growth. Tumor growth rates in the GO-PEG-OSA plus NIR group were inhibited significantly, and the tumor size was just $28 \%$ of that in the GO-PEG-OSA group. In addition, compared to control group, the inhibited tumor growth rate in PTX@GO-PEG-OSA group was approximately $78 \%$, which was $20 \%$ higher than that in the free PTX group. These results indicated that the combination of chemo/PTT/PDT effects induced by PTX@GO-PEG-OSA NSs could kill more tumor cells and showed better antitumor effects than single phototherapy or single chemotherapy. More interestingly, after 9 days of treatment, tumor growth rates in PTX@GO-PEG-OSA plus NIR group were similar to those in the GO-PEG-OSA plus NIR group (Figure $8 \mathrm{C}$ ). The most possible reason for this result was that the killing effect of PTX could not appear in 
that short time. However, over more than 9 days posttreatment, owing to the combination of phototherapy of GO-PEG-OSA NPs and the chemotherapy of PTX, the antitumor effects in the PTX@GO-PEGOSA plus NIR group were more remarkable.

The resected specimens were further sent for histological detection, and tumors were confirmed by H\&E staining (Figure 8D). From TUNEL results, we found that the expression of TUNEL was upregulated in PTX (with or without NIR), GO-PEG-OSA with NIR and PTX@GO-PEG-OSA (with or without NIR) groups, while no evident changes were observed in GO-PEG-OSA and saline plus NIR groups in comparison with saline group. What's more, TUNEL showed highest expression on PTX@GO-PEG-OSA with NIR groups (Figure 8D\&E)[67]. TUNEL, termed as "Terminal deoxynucleotidyl transferase dUTP nick end labeling", is a classic marker to detect DNA fragmentation in apoptotic tumor cells. As is showed highest expression of TUNEL in PTX@GO-PEG-OSA plus NIR group, the results of IHC consistently highlighted its superiority on anti-tumor proliferation.

Enhancing the efficacy of PTX with fewer side effects is a clinical problem being discussed. Body-weight loss was only found in the free PTX and free PTX with NIR groups compared to the other 6 groups (Supplemental materials, Figure S18), revealing that the PTX@GO-PEG-OSA NPs exhibited fewer side effects compared to free PTX NPs in vivo. Collectively, our results provided strong evidences that PTX@GO-PEG-OSA NPs have a promising application for gastric cancer treatment due to their enhanced antitumor effects and less system toxicity.

\section{Conclusions}

In this work, PEG and OSA modified GO NSs were synthesized and loaded with PTX (PTX@GO-PEG-OSA), which showed monodispersion, good biocompatibility and $\mathrm{pH}$-sensitive drug release property. Under proper NIR irradiation (1W/cm²), PTX@GO-PEG-OSA NSs exhibited photo-thermal effect, ROS generation and thermal-accelerated PTX release abilities. From in vitro experiments, PTX@GO-PEG-OSA NSs could efficiently enter PTX-resistant GC cells, reduce their proliferation, increase apoptosis rates and finally induce better antitumor effect compared to free PTX. With NIR-irradiation (1 W/cm², $5 \mathrm{~min})$, PTX@GOPEG-OSA NSs with integration of PDT, mild PTT and chemotherapy exhibited significant super-additive therapeutic effect (called " $1+1>2$ ") by increasing drug sensitivity of PTX-resistant GC cells.

Distinguished from the reported downregulated expression of P-gp, the mechanism of drug resistance's reversal was described that the treatment of PTX@GO-PEG-OSA NSs with NIR-irradiation would cause the depolarization of mitochondrial membrane potential, inhibit the activity of mitochondrial respiratory chain complex enzyme and reduce ATP production. Mitochondrial dysfunction could induce mitoROS burst, which aggravates mitochondrial disorders in turn. Furthermore, it was surprisingly found that hyperthermia might not be the only factor of increasing PTX sensitivity, while PDT induced by ROS also seemed to play a more important role via inducing mitochondrial damage. Since energy supply for P-gp was suppressed, PTX-resistant GC cells were therefore re-sensitive to PTX. In conclusion, the welldesigned PTX@GO-PEG-OSA NSs and combinational chemo/photothermal/photodynamic therapy could 
present an effective approach for reversing GC drug resistance at mild temperature both in vitro and in vivo.

\section{Abbreviations}

GO: Graphene oxide; GC: gastric cancer; ATP: adenosine-triphosphate; PTX: Paclitaxel; P-gp: Pglycoprotein; PEG: polyethylene glycol; OSA: oxidized sodium alginate; NSs: nanosheets; NIR: near infrared;ROS:reactive oxygen species; ETC: electron transport chain; PTT: photothermal; PDT: photodynamic; MDR: multiple drug resistance; DDSs: drug delivery systems; EPR: enhanced permeability and retention effect; MTP: mitochondrial transmembrane potential; NH2-PEG-NH2: polyethylene glycol diamine; SA: sodium alginate; EDC: 1-(3-Dimethylaminopropyl)-3-ethylcarbodiimide hydrochloride; NHS: N-hydroxysuccinimide; LE: loading efficiency; FTIR: Fourier transform infrared; TEM: transmission electron microscopy; AFM: atomic force microscopy; OD: optical density; DMEM: Dulbecco's modified Eagle's medium; FBS: fetal bovine serum; CCK-8: Cell Counting Kit-8 assay; H\&E: hematoxylin and eosin; HGC-27/PTX: PTX-resistant GC cells; UHPLC: ultra-high-performance liquid chromatography; CLSM:

Confocal Laser Scanning Microscopy; EDU: 5-ethinyl-2' DNA nucleoside uracil ; mitoROS: mitochondrial reactive oxygen species; IHC: immunohistochemistry; SDS-PAGE: sodium dodecyl sulfate polyacrylamide gel electrophoresis; ABC: (ATP)-binding cassette; NAC: N-Acetyl-L-cysteine.

\section{Declarations}

\section{Ethics approval and consent to participate}

All the animal experiments were conducted in accordance with the guidelines and the ethical standards of the Institutional Animal Care and Use Committee of the Nanfang Hospital of Southern Medical University.

\section{Consent for publication}

All authors agree to be published.

\section{Availability of data and materials}

The datasets used during the present study are available from the corresponding author upon reasonable request.

\section{Competing interests}

The authors declare that they have no competing interests.

\section{Funding}


This work was supported by grants from National Natural Science Foundation of China ((82001948; 82001298; 81971746), China Postdoctoral Science Foundation (2019M662977; 2019M662986), Natural Science Foundation of Guangdong Province, China (2017A030306023), Science research cultivation program of stomatological hospital, Southern medical university (PY2019006), Guangdong Basic and Applied Basic Research Foundation (2019A1515110635), Guangdong Provincial Major Talents Project (2019JC05Y361), Outstanding Youths Development Scheme of Nanfang Hospital, Southern Medical University (2017J006), Medical Scientific Research Foundation of Guangdong Province of China (A2020131; A2020297) and Special Funds for Cultivation of Guangdong College Students' Scientific and Technological Innovation (pdjh2020a0106; pdjh2020a0109; pdjh2020a0108).

\section{Authors' contributions}

WG, ZC, XF, BZ, GL and YH designed the study. WG, ZC and XF collated the data, carried out data analyses and produced the initial draft of the manuscript. BZ, GL and $\mathrm{YH}$ revised the figures and tables. All authors contributed to drafting the manuscript, read and approved the final manuscript.

\section{Acknowledgements}

The authors want to thank PD Dr. Gengtai Ye from Southern Medical University for technical support.

\section{Authors' information}

${ }^{1}$ Department of General Surgery, Nanfang Hospital, Southern Medical University, Guangzhou 510515, China. ${ }^{2}$ Guangdong Provincial Stomatology Hospital, Southern Medical University, Guangzhou 510000,

China. ${ }^{3}$ Guangdong Provincial Key Laboratory of Cancer Immunotherapy, Guangzhou Key Laboratory of Tumor Immunology Research, Cancer Research Institute, School of Basic Medical Sciences, Southern Medical University, Guangzhou 510515, PR China

\section{References}

1 Bray F, Ferlay J, Soerjomataram I, Siegel RL, Torre LA, Jemal A. Global cancer statistics 2018: GLOBOCAN estimates of incidence and mortality worldwide for 36 cancers in 185 countries. CA: a cancer journal for clinicians. 2018; 68(6): 394-424. https://doi.org/10.3322/caac.21492.

2 Cronin KA, Lake AJ, Scott S, Sherman RL, Noone A-M, Howlader N, Henley SJ, Anderson RN, Firth AU, Ma J, Kohler BA, Jemal A. Annual Report to the Nation on the Status of Cancer, part I: National cancer statistics. Cancer. 2018; 124(13): 2785-2800. https://doi.org/10.1002/cncr.31551.

3 Choi AH, Kim J, Chao J. Perioperative chemotherapy for resectable gastric cancer: MAGIC and beyond. World J Gastroenterol. 2015; 21(24): 7343-7348. https://doi.org/10.3748/wjg.v21.i24.7343.

4 Hu Y, Huang C, Sun Y, Su X, Cao H, Hu J, Xue Y, Suo J, Tao K, He X, Wei H, Ying M, Hu W, Du X, Chen P, Liu H, Zheng C, Liu F, Yu J, Li Z, Zhao G, Chen X, Wang K, Li P, Xing J, Li G. Morbidity and Mortality of 
Laparoscopic Versus Open D2 Distal Gastrectomy for Advanced Gastric Cancer: A Randomized Controlled Trial. Journal of clinical oncology : official journal of the American Society of Clinical Oncology. 2016; 34(12): 1350-1357. https://doi.org/10.1200/JC0.2015.63.7215.

5 Yu J, Huang C, Sun Y, Su X, Cao H, Hu J, Wang K, Suo J, Tao K, He X, Wei H, Ying M, Hu W, Du X, Hu Y, Liu H, Zheng C, Li P, Xie J, Liu F, Li Z, Zhao G, Yang K, Liu C, Li H, Chen P, Ji J, Li G, Chinese Laparoscopic Gastrointestinal Surgery Study G. Effect of Laparoscopic vs Open Distal Gastrectomy on 3-Year DiseaseFree Survival in Patients With Locally Advanced Gastric Cancer: The CLASS-01 Randomized Clinical Trial. JAMA. 2019; 321(20): 1983-1992. https://doi.org/10.1001/jama.2019.5359.

6 Orr GA, Verdier-Pinard P, McDaid H, Horwitz SB. Mechanisms of Taxol resistance related to microtubules. Oncogene. 2003; 22(47): 7280-7295. https://doi.org/10.1038/sj.onc.1206934.

7 Sangrajrang S, Fellous A. Taxol resistance. Chemotherapy. 2000; 46(5): 327-334. https://doi.org/10.1159/000007306.

8 Xu MS, Yang GX, Bi HT, Xu JT, Dong SM, Jia T, Wang Z, Zhao RX, Sun QQ, Gai SL, He F, Yang D, Yang PP. An intelligent nanoplatform for imaging-guided photodynamic/photothermal/chemo-therapy based on upconversion nanoparticles and CuS integrated black phosphorus. Chemical Engineering Journal. 2020; 382: 12. https://doi.org/10.1016/j.cej.2019.122822.

9 Guo W, Deng L, Chen Z, Chen Z, Yu J, Liu H, Li T, Lin T, Chen H, Zhao M, Zhang L, Li G, Hu Y. Vitamin B12-conjugated sericin micelles for targeting CD320-overexpressed gastric cancer and reversing drug resistance. Nanomedicine (London, England). 2019; 14(3): 353-370. https://doi.org/10.2217/nnm-20180321.

10 Liu Z, Robinson JT, Sun X, Dai H. PEGylated nanographene oxide for delivery of water-insoluble cancer drugs. Journal of the American Chemical Society. 2008; 130(33): 10876-10877. https://doi.org/10.1021/ja803688x.

11 Bao H, Pan Y, Ping Y, Sahoo NG, Wu T, Li L, Li J, Gan LH. Chitosan-functionalized graphene oxide as a nanocarrier for drug and gene delivery. Small (Weinheim an der Bergstrasse, Germany). 2011; 7(11): 1569-1578. https://doi.org/10.1002/smll.201100191.

12 Jiang X, Ruan G, Huang Y, Chen Z, Yuan H, Du F. Assembly and application advancement of organicfunctionalized graphene-based materials: A review. Journal of separation science. 2020:

10.1002/jssc.201900694. https://doi.org/10.1002/jssc.201900694.

13 Xia YP, Zhang HZ, Huang PR, Huang CW, Xu F, Zou YJ, Chu HL, Yan EH, Sun LX. Graphene-oxideinduced lamellar structures used to fabricate novel composite solid-solid phase change materials for thermal energy storage. Chemical Engineering Journal. 2019; 362: 909-920.

https://doi.org/10.1016/j.cej.2019.01.097. 
14 Feng X, Chen L, Guo W, Zhang Y, Lai X, Shao L, Li Y. Graphene oxide induces p62/SQSTM-dependent apoptosis through the impairment of autophagic flux and lysosomal dysfunction in PC12 cells. Acta biomaterialia. 2018; 81: 278-292. https://doi.org/10.1016/j.actbio.2018.09.057.

15 Geetha Bai R, Muthoosamy K, Shipton FN, Manickam S. Acoustic cavitation induced generation of stabilizer-free, extremely stable reduced graphene oxide nanodispersion for efficient delivery of paclitaxel in cancer cells. Ultrasonics sonochemistry. 2017; 36: 129-138.

https://doi.org/10.1016/j.ultsonch.2016.11.021.

16 Jia J, Wang CX, Chen KL, Yin YJ. Drug release of yolk/shell microcapsule controlled by pH-responsive yolk swelling. Chemical Engineering Journal. 2017; 327: 953-961.

https://doi.org/10.1016/j.cej.2017.06.170.

17 Gao C, Tang F, Gong G, Zhang J, Hoi MPM, Lee SMY, Wang R. pH-Responsive prodrug nanoparticles based on a sodium alginate derivative for selective co-release of doxorubicin and curcumin into tumor cells. Nanoscale. 2017; 9(34): 12533-12542. https://doi.org/10.1039/c7nr03611f.

$18 \mathrm{Mu}$ B, Lu C, Liu P. Disintegration-controllable stimuli-responsive polyelectrolyte multilayer microcapsules via covalent layer-by-layer assembly. Colloids Surf B Biointerfaces. 2011; 82(2): 385-390. https://doi.org/10.1016/j.colsurfb.2010.09.024.

19 Wang L, Zhou W, Wang Q, Xu C, Tang Q, Yang H. An Injectable, Dual Responsive, and Self-Healing Hydrogel Based on Oxidized Sodium Alginate and Hydrazide-Modified Poly(ethyleneglycol). Molecules. 2018; 23(3). https://doi.org/10.3390/molecules23030546.

20 He YC, Cong C, Liu ZW, Li XL, Zhu RY, Gao DW. Stealth surface driven accumulation of "Trojan Horse" for tumor hypoxia relief in combination with targeted cancer therapy. Chemical Engineering Journal. 2019; 378: 8. https://doi.org/10.1016/j.cej.2019.122252.

21 Li H, Liu C, Zeng YP, Hao YH, Huang JW, Yang ZY, Li R. Nanoceria-Mediated Drug Delivery for Targeted Photodynamic Therapy on Drug-Resistant Breast Cancer. ACS Appl Mater Interfaces. 2016; 8(46): 3151031523. https://doi.org/10.1021/acsami.6b07338.

22 Wang M, Xiao Y, Li Y, Wu J, Li F, Ling D, Gao J. Reactive oxygen species and near-infrared light dualresponsive indocyanine green-loaded nanohybrids for overcoming tumour multidrug resistance. Eur J Pharm Sci. 2019; 134: 185-193. https://doi.org/10.1016/j.ejps.2019.04.021.

23 Li Q, Hong L, Li H, Liu C. Graphene oxide-fullerene C(60) (GO-C(60)) hybrid for photodynamic and photothermal therapy triggered by near-infrared light. Biosens Bioelectron. 2017; 89(Pt 1): 477-482. https://doi.org/10.1016/j.bios.2016.03.072.

24 Sun J, Song L, Fan Y, Tian L, Luan S, Niu S, Ren L, Ming W, Zhao J. Synergistic Photodynamic and Photothermal Antibacterial Nanocomposite Membrane Triggered by Single NIR Light Source. ACS Appl 
Mater Interfaces. 2019; 11(30): 26581-26589. https://doi.org/10.1021/acsami.9b07037.

25 Gao S, Zhang L, Wang G, Yang K, Chen M, Tian R, Ma Q, Zhu L. Hybrid graphene/Au activatable theranostic agent for multimodalities imaging guided enhanced photothermal therapy. Biomaterials. 2016; 79: 36-45. https://doi.org/10.1016/j.biomaterials.2015.11.041.

26 Liao GF, He F, Li Q, Zhong L, Zhao RZ, Che HN, Gao HY, Fang BZ. Emerging graphitic carbon nitridebased materials for biomedical applications. Progress in Materials Science. 2020; 112: 21. https://doi.org/10.1016/j.pmatsci.2020.100666.

27 Le-Tien C, Millette M, Lacroix M, Mateescu M-A. Modified alginate matrices for the immobilization of bioactive agents. Biotechnology and applied biochemistry. 2004; 39(Pt 2): 189-198. https://doi.org/10.1042/BA20030054.

28 Sun X, Zebibula A, Dong X, Zhang G, Zhang D, Qian J, He S. Aggregation-Induced Emission Nanoparticles Encapsulated with PEGylated Nano Graphene Oxide and Their Applications in Two-Photon Fluorescence Bioimaging and Photodynamic Therapy in Vitro and in Vivo. ACS applied materials \& interfaces. 2018; 10(30): 25037-25046. https://doi.org/10.1021/acsami.8b05546.

29 Sun J, Tan H. Alginate-Based Biomaterials for Regenerative Medicine Applications. Materials (Basel). 2013; 6(4): 1285-1309. https://doi.org/10.3390/ma6041285.

30 Yao W, Wang J, Wang P, Wang X, Yu S, Zou Y, Hou J, Hayat T, Alsaedi A, Wang X. Synergistic coagulation of $\mathrm{GO}$ and secondary adsorption of heavy metal ions on $\mathrm{Ca} / \mathrm{Al}$ layered double hydroxides. Environmental pollution (Barking, Essex : 1987). 2017; 229: 827-836.

https://doi.org/10.1016/j.envpol.2017.06.084.

31 Wilfong WC, Kail BW, Bank TL, Howard BH, Gray ML. Recovering Rare Earth Elements from Aqueous Solution with Porous Amine-Epoxy Networks. ACS applied materials \& interfaces. 2017; 9(21): 1828318294. https://doi.org/10.1021/acsami.7b03859.

32 Almeida A, Silva D, Gonçalves V, Sarmento B. Synthesis and characterization of chitosan-graftedpolycaprolactone micelles for modulate intestinal paclitaxel delivery. Drug delivery and translational research. 2018; 8(2): 387-397. https://doi.org/10.1007/s13346-017-0357-8.

33 Luiz MT, Abriata JP, Raspantini GL, Tofani LB, Fumagalli F, de Melo SMG, Emery FdS, Swiech K, Marcato PD, Lee R, Marchetti JM. In vitro evaluation of folate-modified PLGA nanoparticles containing paclitaxel for ovarian cancer therapy. Materials science \& engineering. C, Materials for biological applications. 2019; 105: 110038-110038. https://doi.org/10.1016/j.msec.2019.110038.

34 Chiang M-Y, Lin Y-Z, Chang S-J, Shyu W-C, Lu H-E, Chen S-Y. Direct Reprogramming of Human Suspension Cells into Mesodermal Cell Lineages via Combined Magnetic Targeting and Photothermal 
Stimulation by Magnetic Graphene Oxide Complexes. Small (Weinheim an der Bergstrasse, Germany). 2017; 13(32): 10.1002/smll.201700703. https://doi.org/10.1002/smll.201700703.

35 Liu W, Zhang X, Zhou L, Shang L, Su Z. Reduced graphene oxide (rGO) hybridized hydrogel as a nearinfrared (NIR)/pH dual-responsive platform for combined chemo-photothermal therapy. Journal of colloid and interface science. 2019; 536: 160-170. https://doi.org/10.1016/j.jcis.2018.10.050.

36 Deng R-H, Zou M-Z, Zheng D, Peng S-Y, Liu W, Bai X-F, Chen H-S, Sun Y, Zhou P-H, Zhang X-Z. Nanoparticles from Cuttlefish Ink Inhibit Tumor Growth by Synergizing Immunotherapy and Photothermal Therapy. ACS nano. 2019; 13(8): 8618-8629. https://doi.org/10.1021/acsnano.9b02993.

37 Zhang D, Wei L, Zhong M, Xiao L, Li H-W, Wang J. The morphology and surface charge-dependent cellular uptake efficiency of upconversion nanostructures revealed by single-particle optical microscopy. Chemical science. 2018; 9(23): 5260-5269. https://doi.org/10.1039/c8sc01828f.

38 Tran A-V, Shim K, Vo Thi T-T, Kook J-K, An SSA, Lee S-W. Targeted and controlled drug delivery by multifunctional mesoporous silica nanoparticles with internal fluorescent conjugates and external polydopamine and graphene oxide layers. Acta biomaterialia. 2018; 74: 397-413. https://doi.org/10.1016/j.actbio.2018.05.022.

39 Zhao X, Liu L, Li X, Zeng J, Jia X, Liu P. Biocompatible graphene oxide nanoparticle-based drug delivery platform for tumor microenvironment-responsive triggered release of doxorubicin. Langmuir : the ACS journal of surfaces and colloids. 2014; 30(34): 10419-10429. https://doi.org/10.1021/la502952f.

40 Huang J, Zong C, Shen H, Liu M, Chen B, Ren B, Zhang Z. Mechanism of cellular uptake of graphene oxide studied by surface-enhanced Raman spectroscopy. Small (Weinheim an der Bergstrasse, Germany). 2012; 8(16): 2577-2584. https://doi.org/10.1002/smll.201102743.

41 Merrifield CJ, Kaksonen M. Endocytic accessory factors and regulation of clathrin-mediated endocytosis. Cold Spring Harbor perspectives in biology. 2014; 6(11): a016733-a016733. https://doi.org/10.1101/cshperspect.a016733.

42 Mayor S, Pagano RE. Pathways of clathrin-independent endocytosis. Nature reviews. Molecular cell biology. 2007; 8(8): 603-612. https://doi.org/10.1038/nrm2216.

43 Zhang C, Shi G, Zhang J, Niu J, Huang P, Wang Z, Wang Y, Wang W, Li C, Kong D. Redox- and lightresponsive alginate nanoparticles as effective drug carriers for combinational anticancer therapy. Nanoscale. 2017; 9(9): 3304-3314. https://doi.org/10.1039/c7nr00005g.

44 Mead TJ, Lefebvre V. Proliferation assays (BrdU and EdU) on skeletal tissue sections. Methods in molecular biology (Clifton, N.J.). 2014; 1130: 233-243. https://doi.org/10.1007/978-1-62703-989-5_17.

45 Porter AG, Jänicke RU. Emerging roles of caspase-3 in apoptosis. Cell death and differentiation. 1999; 6(2): 99-104. https://doi.org/10.1038/sj.cdd.4400476. 
46 Feng L, Dong Z, Tao D, Zhang Y, Liu Z. The acidic tumor microenvironment: a target for smart cancer nano-theranostics. National Science Review. 2017; 5(2): 269-286. https://doi.org/10.1093/nsr/nwx062.

47 Zhang X, Xi Z, Machuki JO, Luo J, Yang D, Li J, Cai W, Yang Y, Zhang L, Tian J, Guo K, Yu Y, Gao F. Gold Cube-in-Cube Based Oxygen Nanogenerator: A Theranostic Nanoplatform for Modulating Tumor Microenvironment for Precise Chemo-Phototherapy and Multimodal Imaging. ACS Nano. 2019; 13(5): 5306-5325. https://doi.org/10.1021/acsnano.8b09786.

48 Huang J, Huang $Y$, Xue Z, Zeng S. Tumor microenvironment responsive hollow mesoporous $\mathrm{Co}(9) S(8) @ M n O(2)-I C G / D O X$ intelligent nanoplatform for synergistically enhanced tumor multimodal therapy. Biomaterials. 2020; 262: 120346. https://doi.org/10.1016/j.biomaterials.2020.120346.

49 Gottesman MM, Fojo T, Bates SE. Multidrug resistance in cancer: role of ATP-dependent transporters. Nature reviews. Cancer. 2002; 2(1): 48-58. https://doi.org/10.1038/nrc706.

50 Li W, Zhang H, Assaraf YG, Zhao K, Xu X, Xie J, Yang D-H, Chen Z-S. Overcoming ABC transportermediated multidrug resistance: Molecular mechanisms and novel therapeutic drug strategies. Drug resistance updates : reviews and commentaries in antimicrobial and anticancer chemotherapy. 2016; 27: 14-29. https://doi.org/10.1016/j.drup.2016.05.001.

51 Ding Y, Du C, Qian J, Dong C-M. NIR-Responsive Polypeptide Nanocomposite Generates NO Gas, Mild Photothermia, and Chemotherapy to Reverse Multidrug-Resistant Cancer. Nano letters. 2019; 19(7): 43624370. https://doi.org/10.1021/acs.nanolett.9b00975.

52 Yang G, Tian J, Chen C, Jiang D, Xue Y, Wang C, Gao Y, Zhang W. An oxygen self-sufficient NIRresponsive nanosystem for enhanced PDT and chemotherapy against hypoxic tumors. Chemical science. 2019; 10(22): 5766-5772. https://doi.org/10.1039/c9sc00985j.

53 Szakács G, Paterson JK, Ludwig JA, Booth-Genthe C, Gottesman MM. Targeting multidrug resistance in cancer. Nature reviews. Drug discovery. 2006; 5(3): 219-234. https://doi.org/10.1038/nrd1984.

54 Klingenberg M. The ADP-ATP translocation in mitochondria, a membrane potential controlled transport. The Journal of membrane biology. 1980; 56(2): 97-105. https://doi.org/10.1007/bf01875961.

55 Ahn CS, Metallo CM. Mitochondria as biosynthetic factories for cancer proliferation. Cancer \& metabolism. 2015; 3(1): 1-1. https://doi.org/10.1186/s40170-015-0128-2.

56 Lusvarghi S, Ambudkar SV. ATP-dependent thermostabilization of human P-glycoprotein (ABCB1) is blocked by modulators. The Biochemical journal. 2019; 476(24): 3737-3750. https://doi.org/10.1042/BCJ20190736.

57 Letts JA, Sazanov LA. Clarifying the supercomplex: the higher-order organization of the mitochondrial electron transport chain. Nature structural \& molecular biology. 2017; 24(10): 800-808. https://doi.org/10.1038/nsmb.3460. 
58 Balsa E, Marco R, Perales-Clemente E, Szklarczyk R, Calvo E, Landázuri MO, Enríquez JA. NDUFA4 is a subunit of complex IV of the mammalian electron transport chain. Cell metabolism. 2012; 16(3): 378-386. https://doi.org/10.1016/j.cmet.2012.07.015.

59 Zhao R-Z, Jiang S, Zhang L, Yu Z-B. Mitochondrial electron transport chain, ROS generation and uncoupling (Review). International journal of molecular medicine. 2019; 44(1): 3-15. https://doi.org/10.3892/ijmm.2019.4188.

60 Murphy MP. How mitochondria produce reactive oxygen species. The Biochemical journal. 2009; 417(1): 1-13. https://doi.org/10.1042/BJ20081386.

61 Bhandary B, Marahatta A, Kim H-R, Chae H-J. An involvement of oxidative stress in endoplasmic reticulum stress and its associated diseases. International journal of molecular sciences. 2012; 14(1): 434-456. https://doi.org/10.3390/ijms14010434.

62 Loreto C, La Rocca G, Anzalone R, Caltabiano R, Vespasiani G, Castorina S, Ralph DJ, Cellek S, Musumeci G, Giunta S, Djinovic R, Basic D, Sansalone S. The role of intrinsic pathway in apoptosis activation and progression in Peyronie's disease. BioMed research international. 2014; 2014: 616149616149. https://doi.org/10.1155/2014/616149.

63 He F, Ji HJ, Feng LL, Wang Z, Sun QQ, Zhong CN, Yang D, Gai SL, Yang PP, Lin J. Construction of thiolcapped ultrasmall Au-Bi bimetallic nanoparticles for X-ray CT imaging and enhanced antitumor therapy efficiency. Biomaterials. 2021; 264: 13. https://doi.org/10.1016/j.biomaterials.2020.120453.

64 Dong S, Dong Y, Jia T, Liu S, Liu J, Yang D, He F, Gai S, Yang P, Lin J. GSH-Depleted Nanozymes with Hyperthermia-Enhanced Dual Enzyme-Mimic Activities for Tumor Nanocatalytic Therapy. Adv Mater. 2020; 32(42): e2002439. https://doi.org/10.1002/adma.202002439.

65 He Y, Guo S, Wu L, Chen P, Wang L, Liu Y, Ju H. Near-infrared boosted ROS responsive siRNA delivery and cancer therapy with sequentially peeled upconversion nano-onions. Biomaterials. 2019; 225: 119501119501. https://doi.org/10.1016/j.biomaterials.2019.119501.

66 Deng T, Zhao H, Shi M, Qiu Y, Jiang S, Yang X, Zhao Y, Zhang Y. Photoactivated Trifunctional Platinum Nanobiotics for Precise Synergism of Multiple Antibacterial Modes. Small (Weinheim an der Bergstrasse, Germany). 2019; 15(46): e1902647-e1902647. https://doi.org/10.1002/smll.201902647.

67 Majtnerová P, Roušar T. An overview of apoptosis assays detecting DNA fragmentation. Molecular biology reports. 2018; 45(5): 1469-1478. https://doi.org/10.1007/s11033-018-4258-9.

\section{Figures}




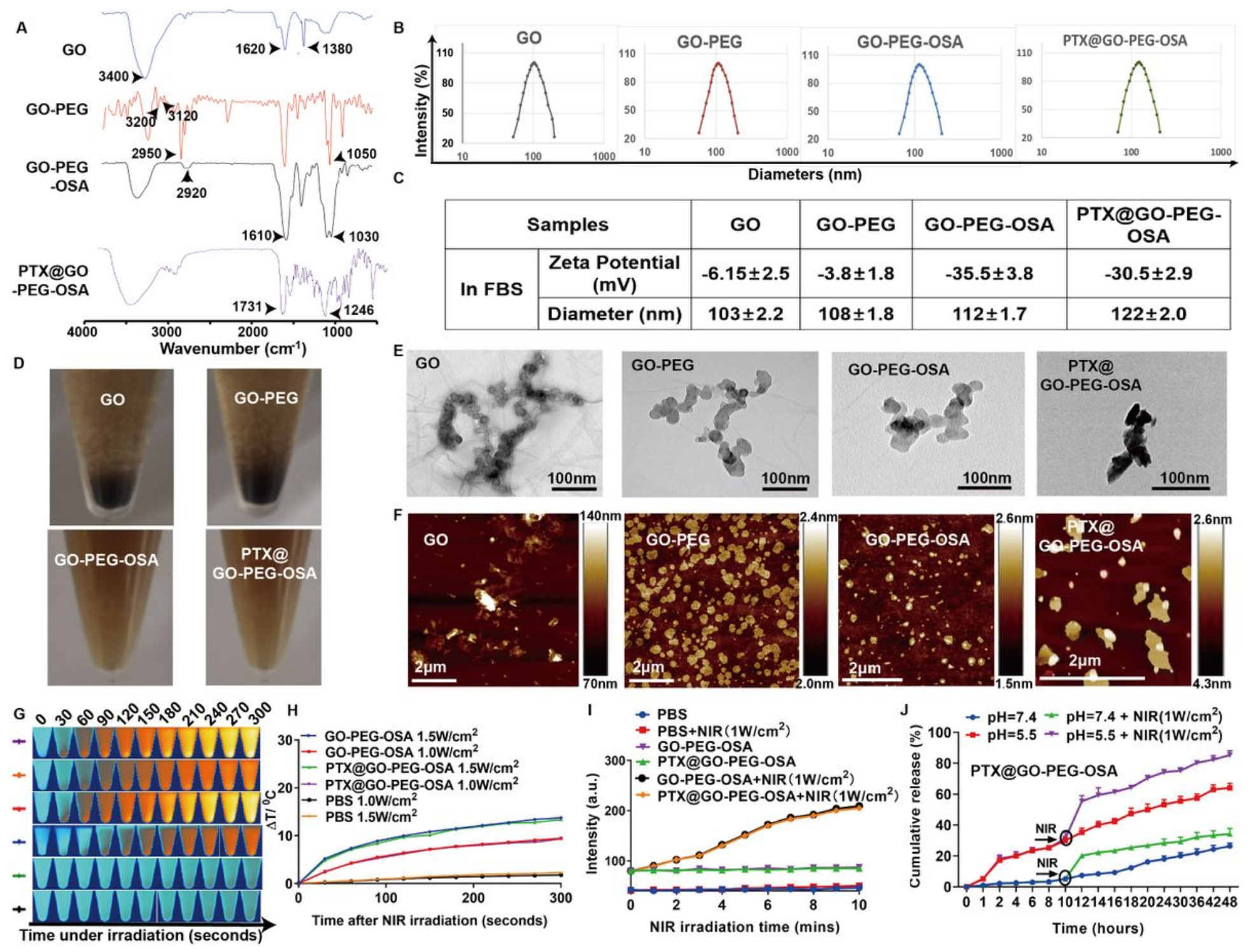

\section{Figure 1}

Physicochemical characteristics of PTX@GO-PEG-OSA NSs. (A) FT-IR spectra of GO, GO-PEG, GO-PEGOSA and PTX@GO-PEG-OSA NSs. (B) Size distribution of GO, GO-PEG, GO-PEG-OSA and PTX@GO-PEGOSA NSs in FBS. (C) Zeta potential and diameters (down) of GO, GO-PEG, GO-PEG-OSA and PTX@GOPEG-OS NSs in FBS. (D) The images of GO, GO-PEG, GO-PEG-OSA and PTX@GO-PEG-OSA NSs after storage in FBS for one month. (E) TEM images of GO, GO-PEG, GO-PEG-OSA and PTX@GO-PEG-OSA NSs. (F) AFM images of GO-PEG-OSA and PTX@GO-PEG-OSA NSs. (G\&H) The temperature curves induced by GO-PEG-OSA, PTX@GO-PEG-OSA NSs and PBS under NIR irradiation. (I) Singlet oxygen generation abilities of GO-PEG-OSA and PTX@GO-PEG-OSA in water, with or without NIR irradiation. (J) Drug release curve for PTX@GO-PEG-OSA NSs. Data are shown as the mean \pm SD, $n=3$. 
A

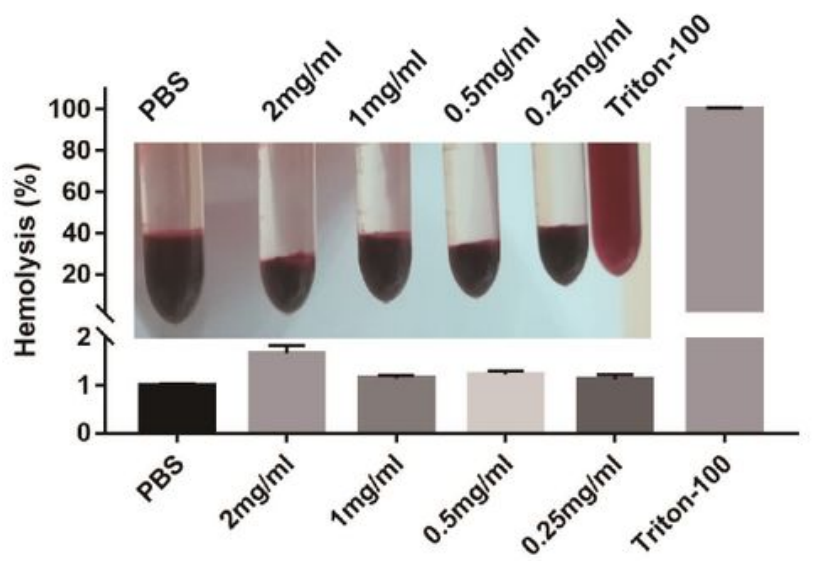

Concentration of GO-PEG-OSA (mg. mL-1)

C
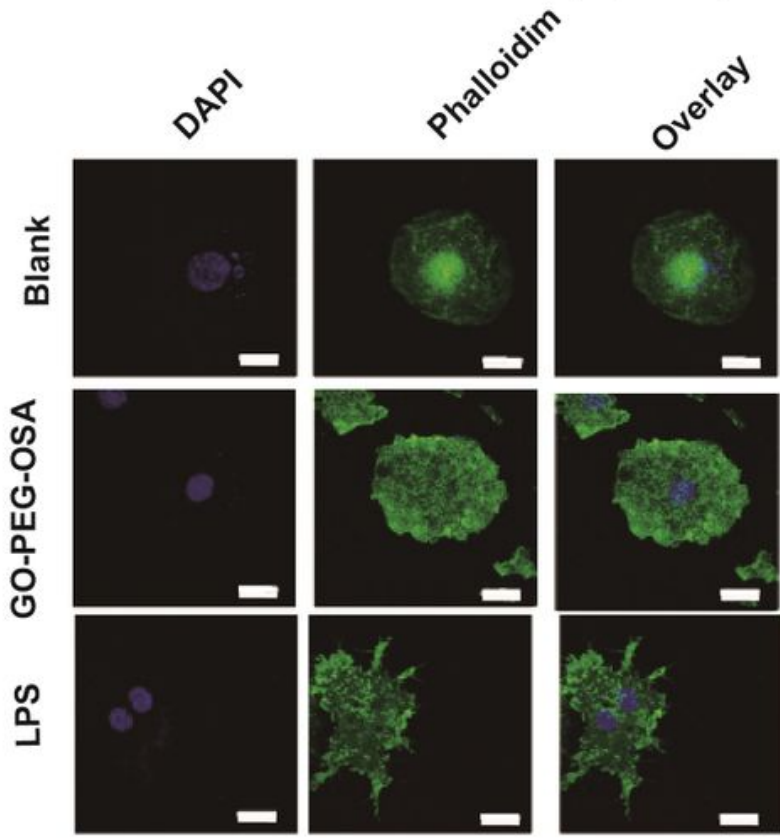

E

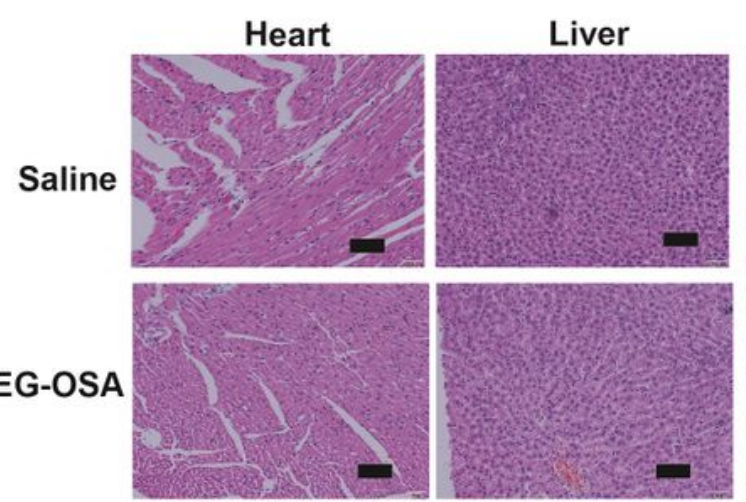

B

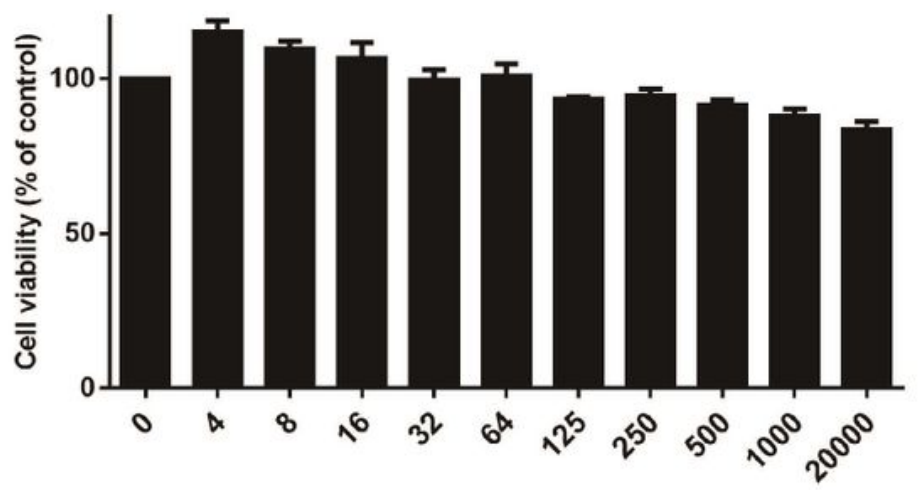

Concentration of GO-PEG-OSA ( $\mu \mathrm{g} . \mathrm{mL}-1$ )
D
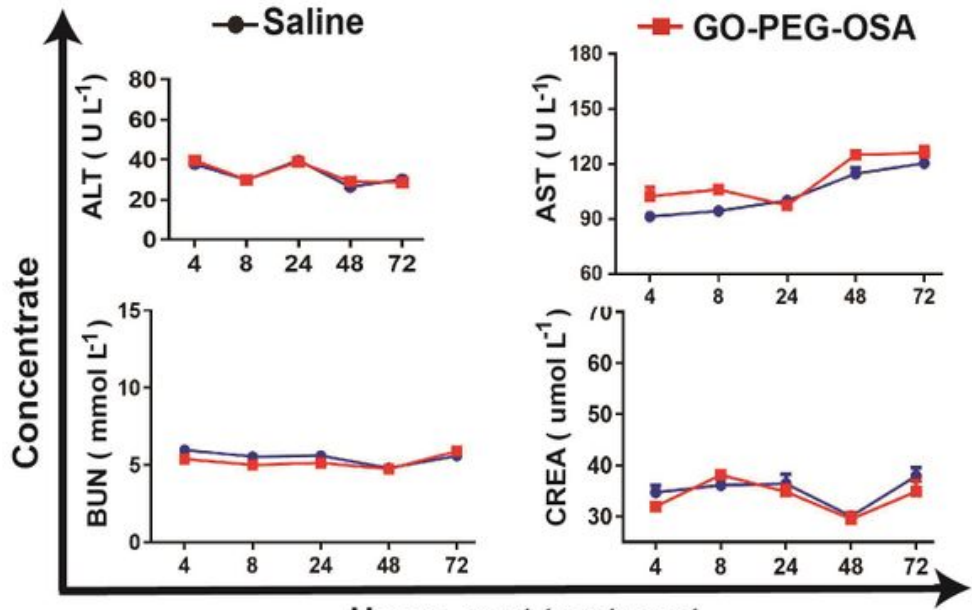

Hours post treatment

Lung

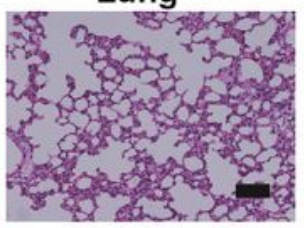

Kidney

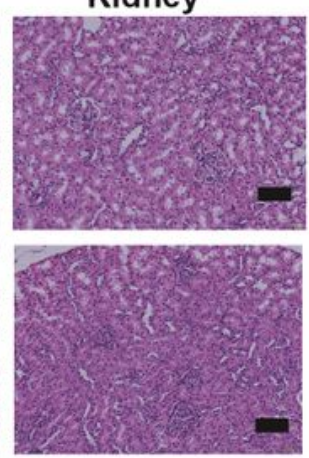

Brain

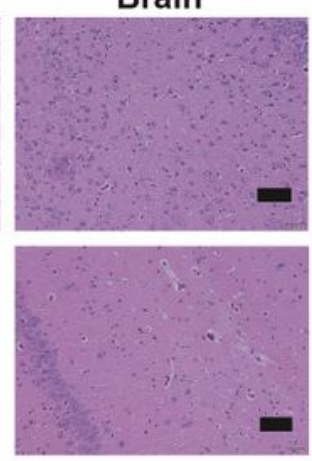

\section{Figure 2}

In vitro and in vivo biocompatibility of GO-PEG-OSA nanosheets. (A) Hemolysis assay results of GO-PEGOSA NSs at different concentrations. Triton X-100 and PBS were used as positive and negative controls, respectively. (B) Cell viabilities of GES-1 cells after incubation with GO-PEG-OSA NSs at different concentrations. (C) Confocal microscopy images showing the morphologies of RAW264.7 cells before (control) and after treatment with LPS or GO-PEG-OSA NSs for $24 \mathrm{~h}$. The F-actin and nuclei were stained 
with the Rhodamine-phalloidin (green) and DAPI (blue), respectively. Scale bar: $20 \mu \mathrm{m}$. (D) Biochemical assay results of the sera collected from SD rats treated with saline or GO-PEG-OSA NSs. AST, aspartate aminotransferase; ALT, alanine aminotransferase; BUN, blood urea nitrogen; UA, blood uric acid. (E) Histopathological analysis of major organ tissues isolated from SD rats treated with saline or GO-PEGOSA NSs. Scale bar, $50 \mu \mathrm{m}$. Data are shown as the mean $\pm \mathrm{SD}, \mathrm{n}=5$.
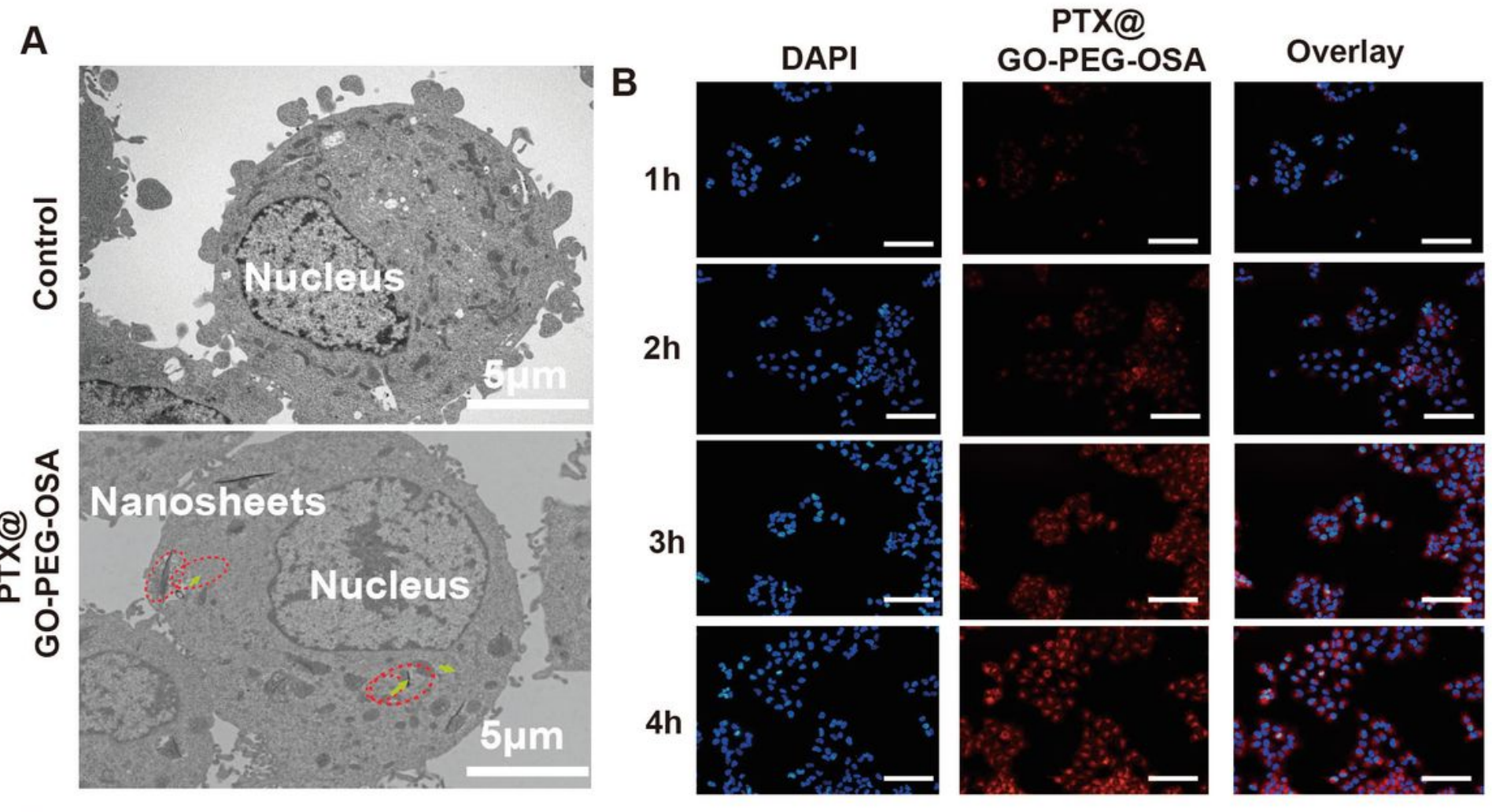

C

D PTX@
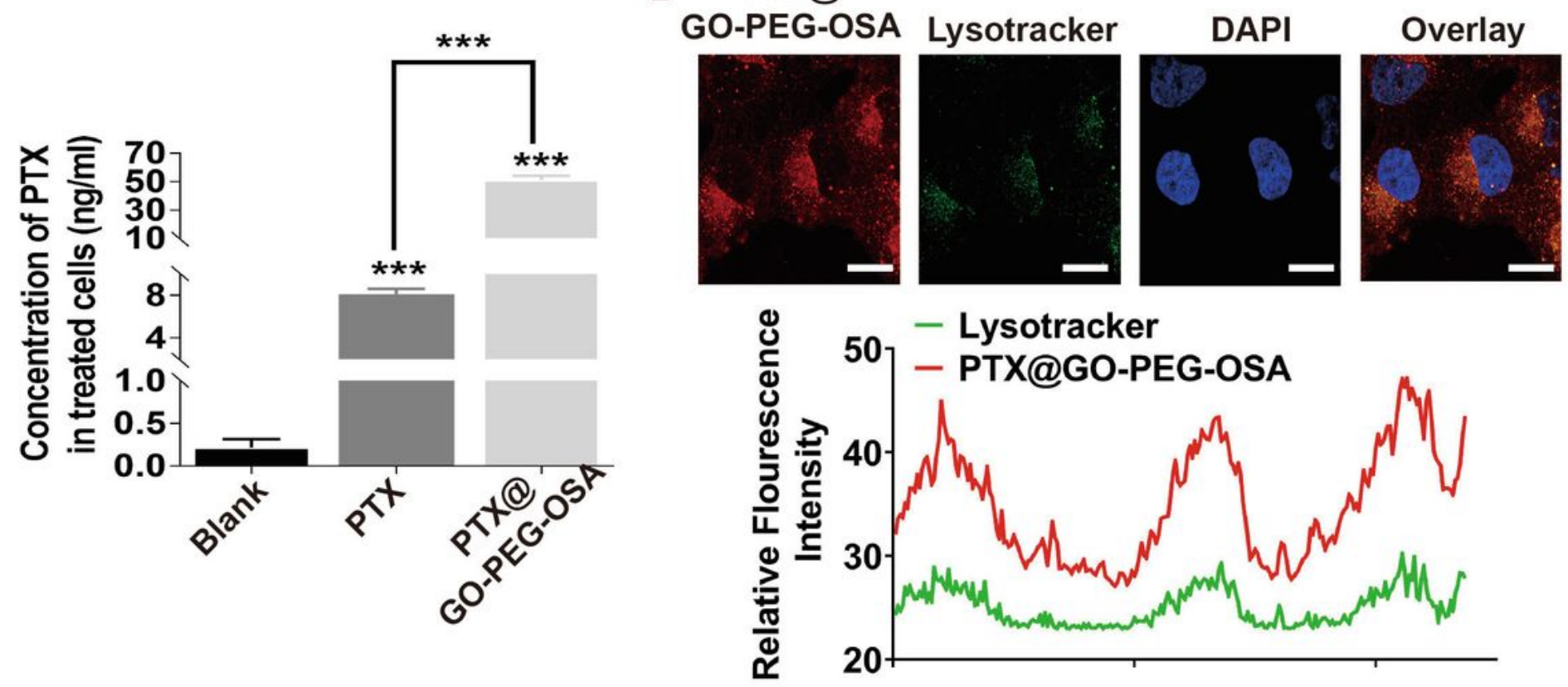

Distance

Figure 3 
Cell uptake of PTX@GO-PEG-OSA NSs (the concentration of PTX was $8 \mu \mathrm{g} / \mathrm{mL}$ ). (A) Bio-TEM images of HGC-27/PTX cells, after incubation with DMEM plus 10\% FBS (control) or PTX@GO-PEG-OSA NSs for 48 hours. Yellow arrows represent PTX@GO-PEG-OSA NSs. (B) Inversed fluorescent microscopy images of HGC-27/PTX cells incubated with GO-PEG-OSA NSs for 1, 2, 3 and 4 hours. Scale bar, $50 \mu \mathrm{m}$. (C) Concentration of PTX in the treated cells determined using UHPLC. (D) CLSM images (up) and fluorescence intensity correlation plot (down) of HGC-27/PTX cells incubated with GO-PEG-OSA NSs for 24 hours. Scale bar, $500 \mathrm{~nm}$. Data are shown as the mean $\pm S D, n=3$.

A

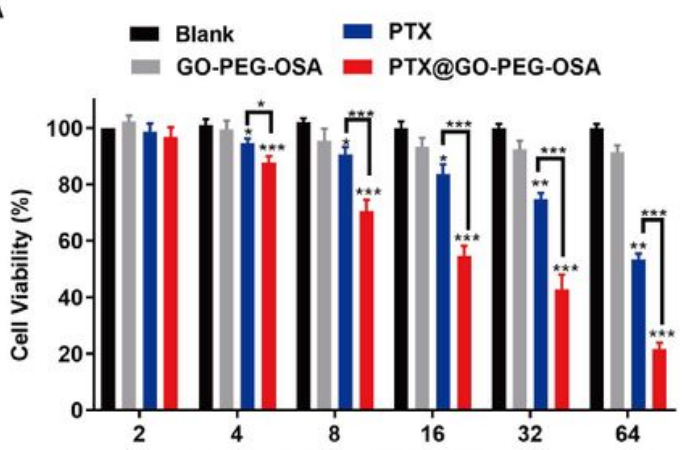

B

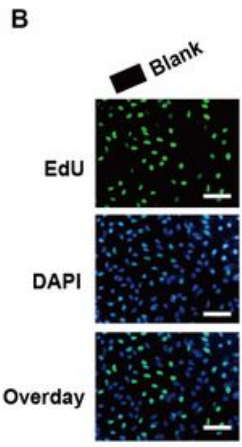

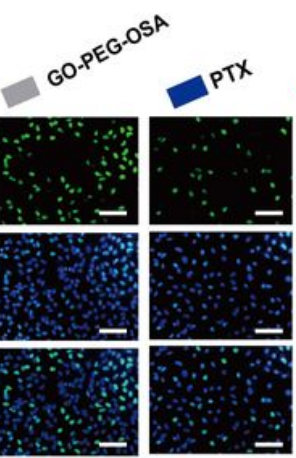

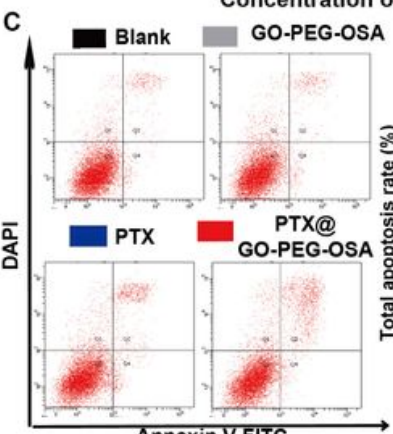

Annexin V-FITC

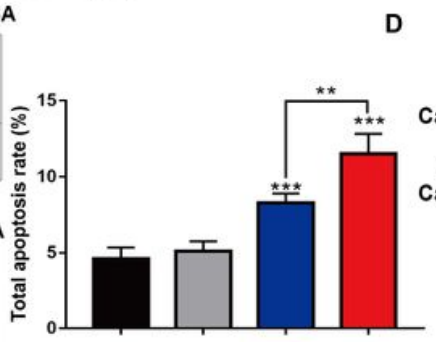

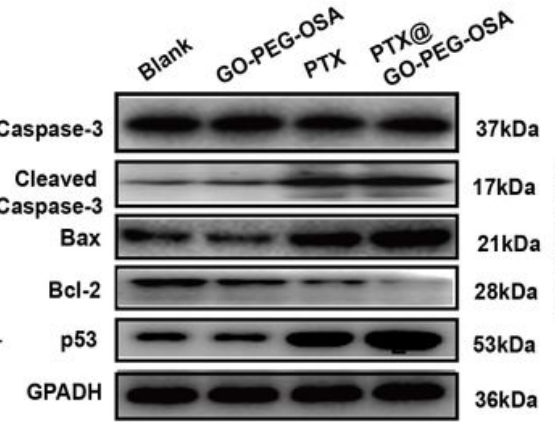
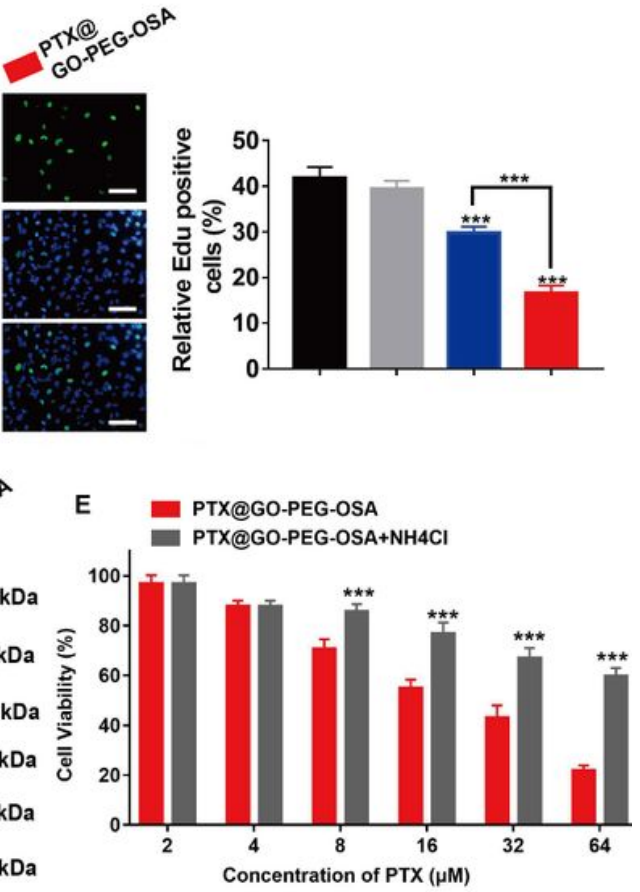

Figure 4

In vitro antitumor effect of PTX@GO-PEG-OSA NSs. (A) Viabilities of HGC-27/PTX cells after incubation with free PTX, PTX@GO-PEG-OSA and GO-PEG-OSA NSs, while the DMEM with 10\% FBS was used as the negative control (blank) group. (B) EdU images and statistics EdU-positive number of HGC-27/PTX cells after the incubation with PTX, PTX@GO-PEG-OSA and GO-PEG-OSA NSs for 48 hours. Scale bar, $100 \mu \mathrm{m}$. (C) Flow cytometry images and statistics apoptotic number of HGC-27/PTX cells after the incubation with PTX, PTX@GO-PEG-OSA and GO-PEG-OSA NSs for 48 hours. (D) Expression of caspase-3, cleaved caspase-3, Bax, Bcl-2, p53 proteins and GAPDH protein in HGC-27/PTX cells after the incubation with PTX, PTX@GO-PEG-OSA and GO-PEG-OSA NSs for 48 hours. (E) Viabilities of HGC-27/PTX cells, with or without treatment by $\mathrm{NH} 4 \mathrm{Cl}$, with the presence of PTX@GO-PEG-OSA NSs for 48 hours. Data are shown as the mean $\pm S D, n=3$. ** indicates $P<0.01$, *** indicates $P<0.001$. 
A

$\begin{array}{ll}\text { Blank } & \text { NIR } \\ \text { GO-PEG-OSA } & \text { GO-PEG-OSA+NIR } \\ \text { PTX@GO-PEG-OSA } & \text { PTX@GO-PEG-OSA+NIR }\end{array}$

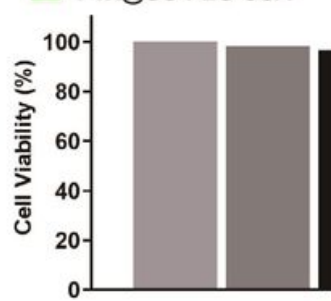

C GO-PEG-OSA

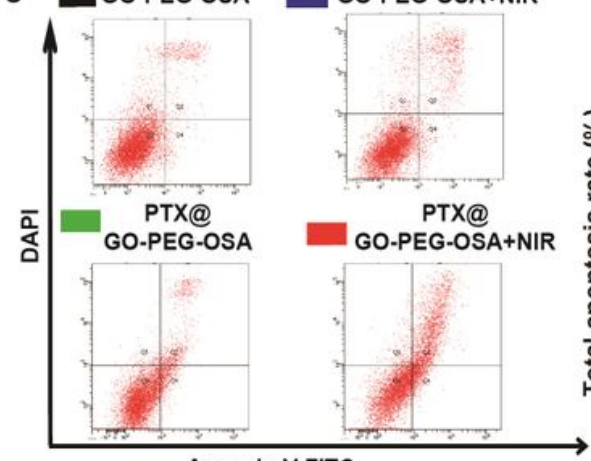

Annexin V-FITC
B
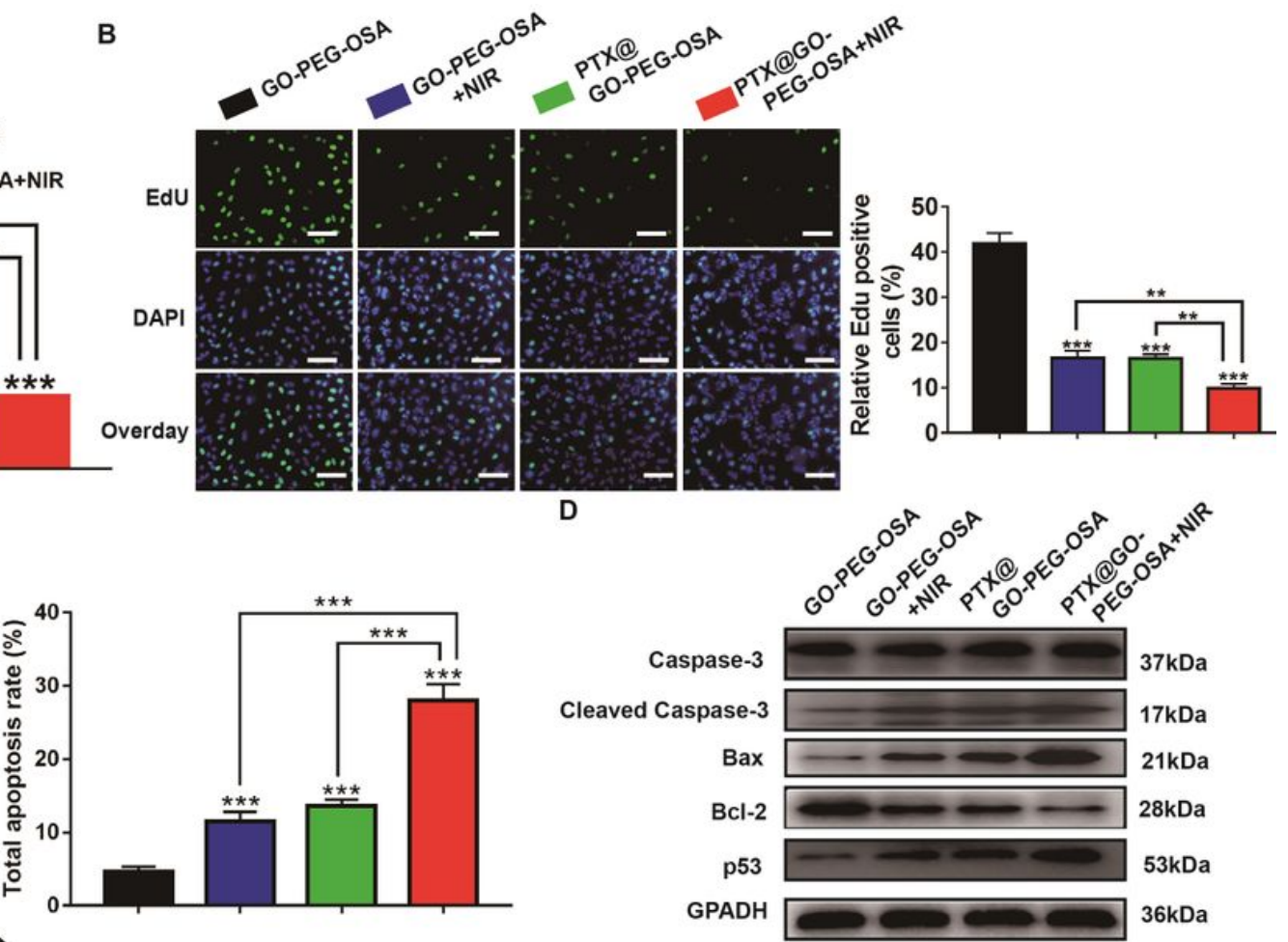

Figure 5

In vitro chemo/photothermal therapeutic effect of PTX@GO-PEG-OSA NSs. (A) Viabilities of HGC-27/PTX cells after incubation with DMEM plus 10\%FBS, DMEN plus 10\% PBS and NIR, GO-PEG-OSA and PTX@GO-PEG-OSA, with or without NIR irradiation. (B) EdU images and statistics EdU-positive number of HGC-27/PTX cells after the incubation with GO-PEG-OSA and PTX@GO-PEG-OSA, with or without NIR irradiation. Scale bar, $100 \mu \mathrm{m}$. (C) Flow cytometry images and statistics apoptotic number of HGC27/PTX cells after the incubation with GO-PEG-OSA and PTX@GO-PEG-OSA, with or without NIR irradiation. (D) Expression of caspase-3, cleaved caspase-3, Bax, Bcl-2, p53 proteins and GAPDH protein in HGC-27/PTX cells after the incubation with GO-PEG-OSA and PTX@GO-PEG-OSA, with or without NIR irradiation. Data are shown as the mean $\pm S D, n=3$. ** indicates $P<0.01$, *** indicates $P<0.001$. 


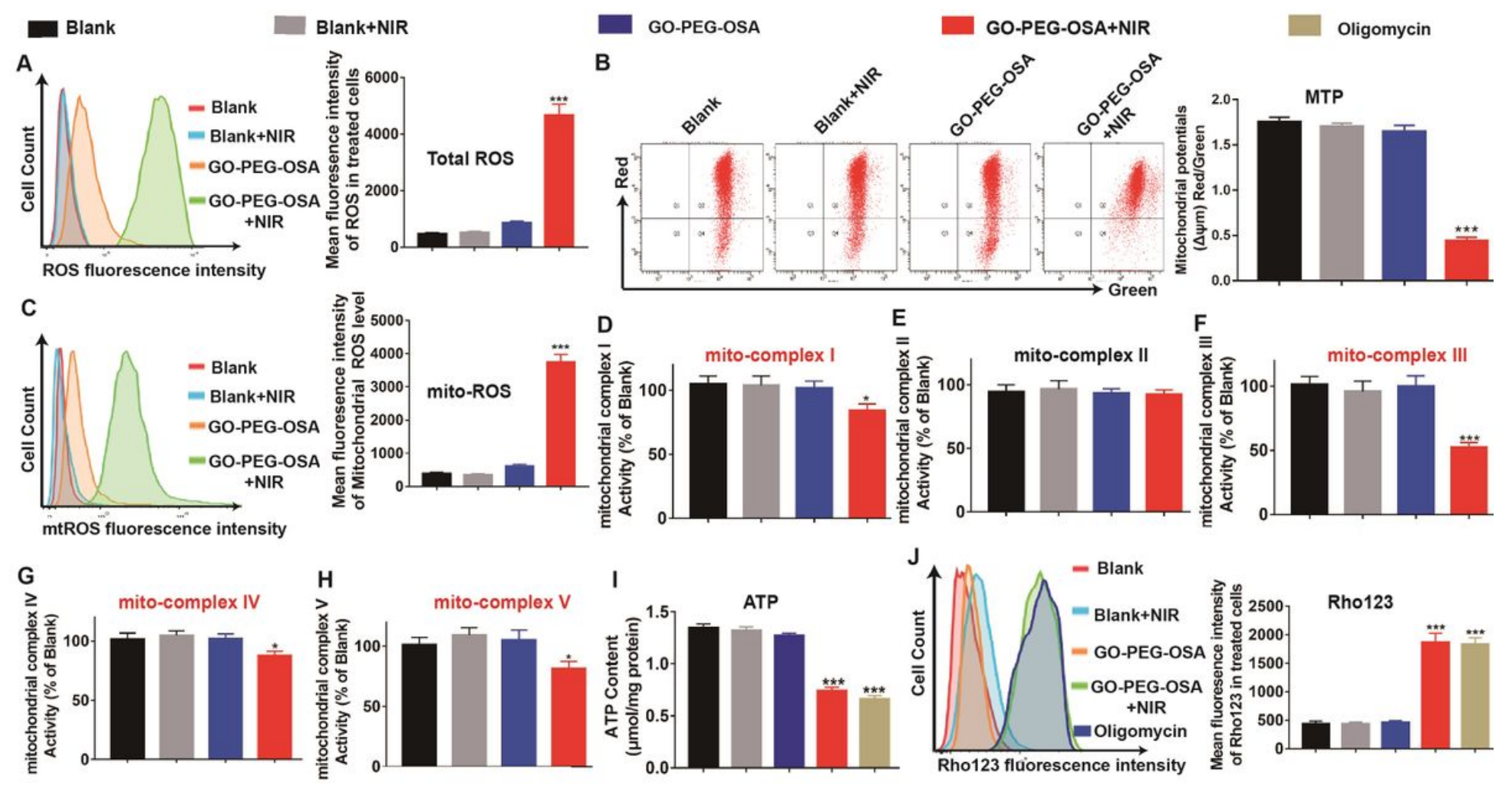

\section{Figure 6}

Production of ROS in mitochondria induced by photo-therapeutic effects of GO-PEG-OSA could combat drug resistance. (A). ROS in HGC-27/PTX cells after incubation with or without NIR in the presence of GOPEG-OSA. (B) MTP in HGC-27/PTX cells after incubation with or without NIR in the presence of GO-PEGOSA. (C) Mitochondrial-derived ROS in HGC-27/PTX cells after incubation with or without NIR in the presence of GO-PEG-OSA. (D-H) Mitochondrial complex I-V activities in HGC-27/PTX cells after incubation with or without NIR in the presence of GO-PEG-OSA. (I) Relative content of ATP in HGC-27/PTX cells after incubation with or without NIR in the presence of GO-PEG-OSA. (J) Relative Rho123 withheld in HGC27/PTX cells after incubation with or without NIR in the presence of GO-PEG-OSA. Data are shown as the mean $\pm S D, n=5$. * indicates $P<0.05$, *** indicates $P<0.001$. 


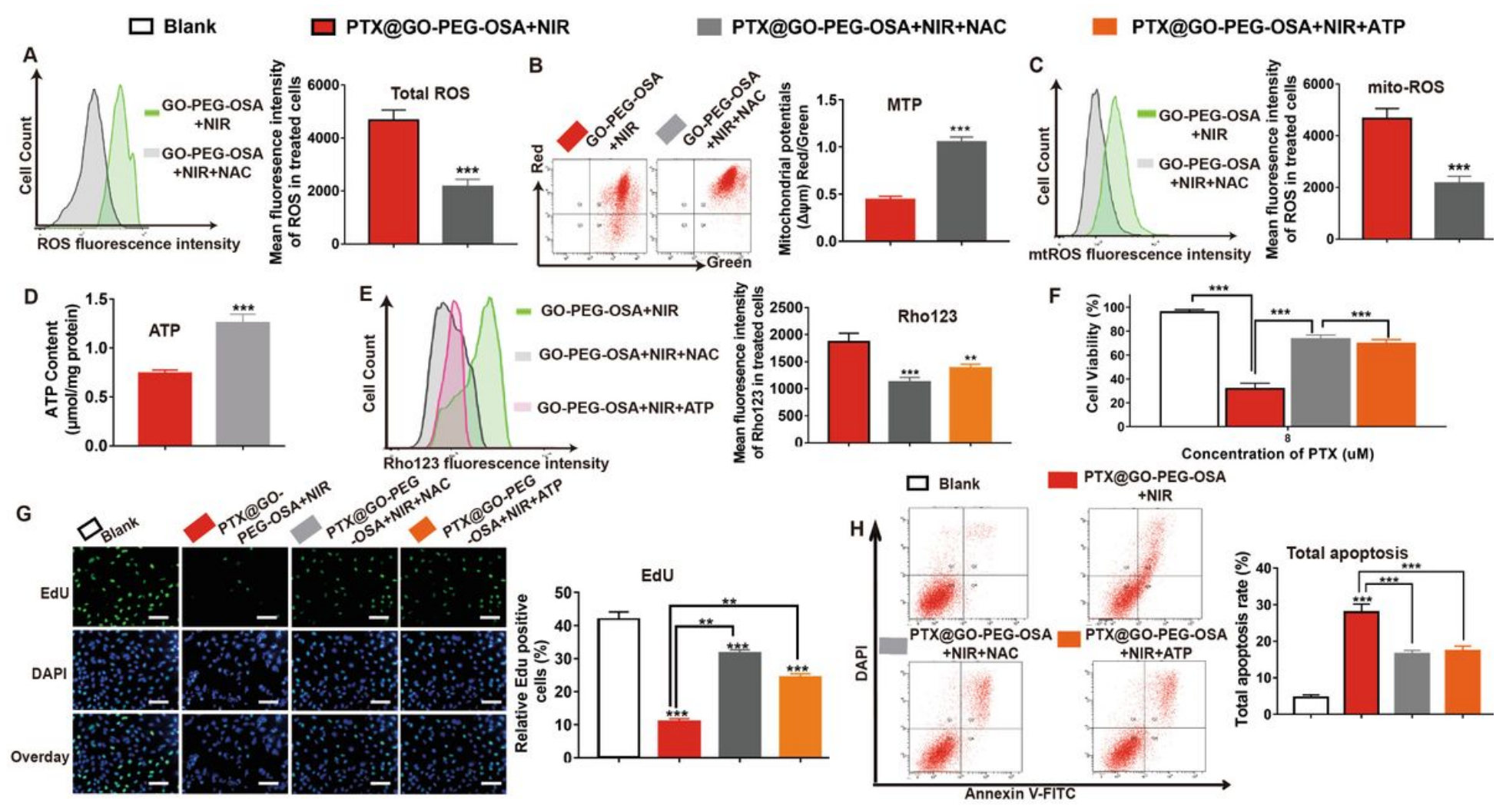

Figure 7

Anti-oxidant (NAC) could increase ATP supply for P-gp efflux pumps and compromise MDR-reversal abilities of GO-PEG-OSA in HGC-27/PTX cells. (A). ROS in GO-PEG-OSA plus NIR-treated HGC-27/PTX cells, with addition of NAC. (B) MTP GO-PEG-OSA plus NIR-treated HGC-27/PTX cells, with addition of NAC. (C) Mitochondrial-derived ROS in GO-PEG-OSA plus NIR-treated HGC-27/PTX cells, with addition of NAC. (D) Relative content of ATP in GO-PEG-OSA plus NIR-treated HGC-27/PTX cells, with addition of NAC. (E) Relative Rho123 withheld in GO-PEG-OSA plus NIR-treated HGC-27/PTX cells, with addition of NAC or exogenous ATP. (F) Viabilities of PTX@GO-PEG-OSA-treated HGC-27/PTX cells, with addition of NAC or exogenous ATP. (G) EdU images and statistics EdU-positive number of PTX@GO-PEG-OSA-treated HGC-27/PTX cells, with addition of NAC or exogenous ATP. Scale bar, $100 \mu \mathrm{m}$. (H) Flow cytometry images and statistics apoptotic number of PTX@GO-PEG-OSA-treated HGC-27/PTX cells, with addition of NAC or exogenous ATP. Data are shown as the mean $\pm S D, n=5$. ** indicates $P<0.01$. *** indicates $P<0.001$. 

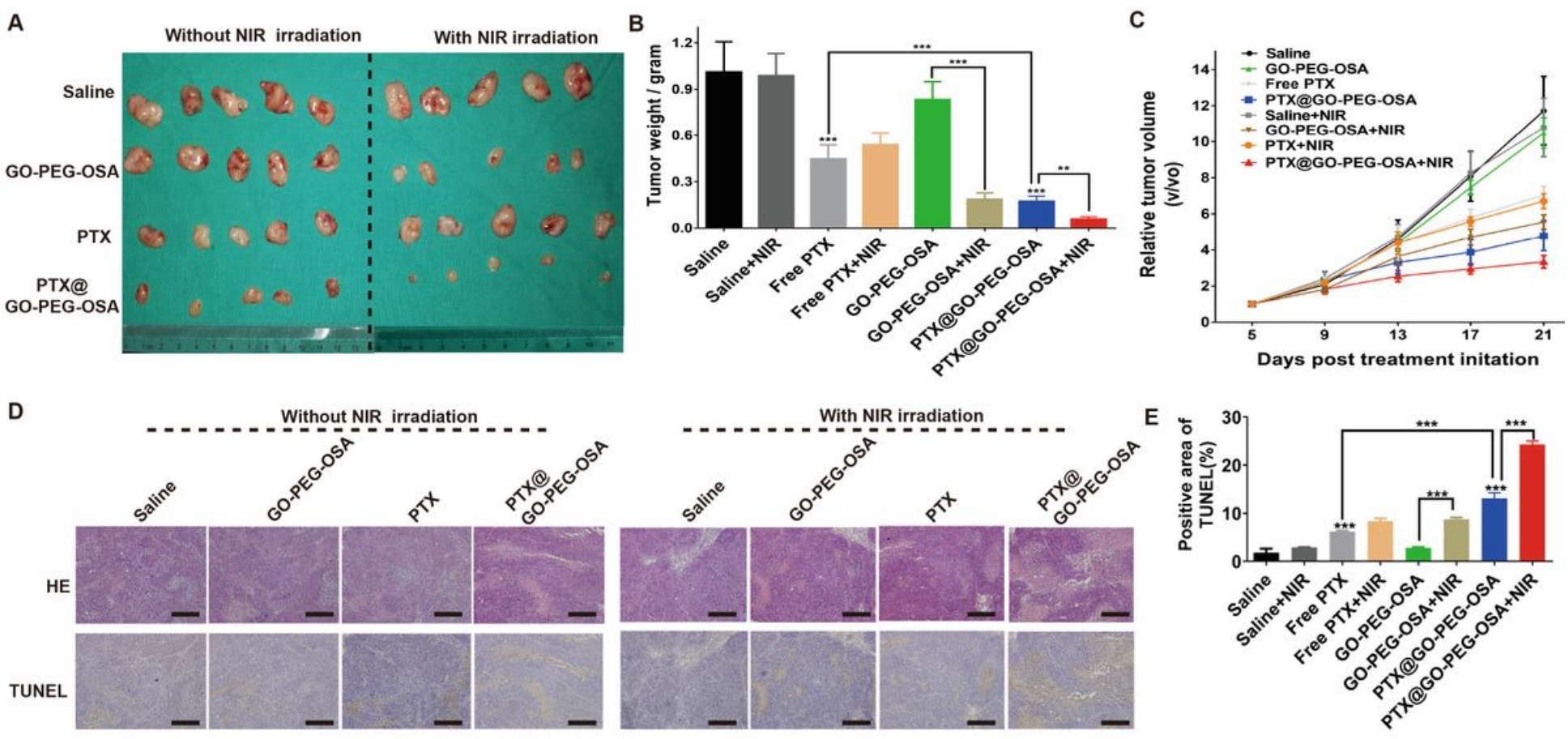

\section{Figure 8}

In vivo photothermal and chemotherapeutic effects of PTX@GO-PEG-OSA NSs. (A) Images of tumors derived from mice treated with drugs. (B) Weight of tumors isolated from mice. (C) Relative volume of tumors isolated from mice. (D) HE and TUNEL images of tumors isolated from mice. (E) The relative expression of TUNEL markers in tumors isolated from mice. Data are shown as the mean $\pm S D, n=5$. Scale bar, $50 \mu \mathrm{m} .{ }^{*} \mathrm{P}<0.05,{ }^{* *} \mathrm{P}<0.001$.

\section{Supplementary Files}

This is a list of supplementary files associated with this preprint. Click to download.

- scheme1.jpg

- scheme2.jpg

- Supplementarymaterials.doc 